\title{
The skirted island: The effect of topography on the flow around planetary scale islands
}

\author{
by Joseph Pedlosky ${ }^{1,2}$, Roberto Iacono ${ }^{3}$, Ernesto Napolitano ${ }^{3}$ and Karl Helfrich ${ }^{1}$
}

\begin{abstract}
The flow around planetary scale islands is examined when the island possesses a topographic skirt representing a steep continental shelf. The model is barotropic and governed by the shallow water equations and the motion is driven by a wind stress with a constant curl. The presence of the strong topographic "skirt" around the island vitiates the elegant Island Rule of Godfrey and the closed potential vorticity contours around the island produced by the topography allow a geostrophic, stationary mode to resonate with an amplitude that is limited only by dissipation. In the limit of weak forcing the outline of the outermost closed potential vorticity isoline essentially replaces the island shape and determines the flow beyond that contour. Stronger nonlinearity produces substantial changes in the flow pattern as well as the transports trapped on the closed contours and the transport between the island and the basin boundary. Laboratory experiments, numerical calculations and analytical results are presented describing the structure of the flow.

A western standing meander at the edge of the island's topography involves a rapid change in the direction of flow and this feature, predicted by analytical and numerical calculations is confirmed in laboratory experiments.

As the measure of nonlinearity is increased beyond a threshold that depends on the ratio of the inertial boundary layer thickness to the Munk layer thickness the flow becomes time dependent and a strong eddy field emerges.

The transports on the closed contours and the inter-basin exchange outside the closed potential vorticity contours show an enhancement over the linear analytical approximation as nonlinearity increases.
\end{abstract}

\section{Introduction}

Ever since the pioneering work of Godfrey (1989) considerable effort has gone into clarifying the role of planetary scale islands in the dynamics of the ocean circulation. Both its intrinsic fluid mechanical interest as well as its application to important ocean circulation questions and its role in climate (e.g. the flow from the Pacific to the Indian Ocean as part of the global ocean overturning circulation) have motivated continued investigation of the phenomenon. There have been numerous studies following Godfrey's.

1. Department of Physical Oceanography, Clark Laboratory, Woods Hole Oceanographic Institution, Woods Hole, Massachusetts, 02543, U.S.A.

2. Corresponding author: email: jpedlosky@whoi.edu

3. ENEA, C. R. Casaccia, Rome, Italy. 
For example, Pedlosky et al. (1997, hereafter PPSH) examined the circulation of islands in various configurations in both linear and nonlinear parameter ranges with analytical, numerical and laboratory models. That study was also motivated by the question of the role of mid-ocean ridge systems, with their interruptions by transform faults that render the ridge a series of disconnected "deep islands."

Studies by Joyce and Proshutinsky (2007) and Nof (2000) have further applied the so-called Island Rule, Godfrey's recipe for determining the circulation around planetary islands in the linear limit. Firing et al. (1999) have applied a form of the rule to the discussion of time-varying flows. Each of these studies has idealized the island as a cylindrical region, i.e. an interior "hole" in the oceanic basin whose boundaries are vertical walls. The hole renders the basin multiply connected and the Island Rule must be used to determine the circulation, or a fluid mechanical equivalent, around the island.

In this paper we take up the question of the effect of topography in the region bounding the island. There have been other studies including isolated topographic features, usually in the context of the oceanic abyssal circulation. For example, Katsman (2006) has investigated the dynamics of the interaction of localized mixing, parameterized as a localization of an upwelling velocity forcing an abyssal flow with an isolated ridge in the context of the abyssal circulation. Huang and Jin (2002) examined the role of the mid-Atlantic ridge and associated variations in mixing rate in a baroclinic model in altering the classic StommelArons (1960) picture of the abyssal circulation. In each of these cases the emphasis was primarily on the local effect of topography on the circulation. In the following study we will also emphasize the basin-wide effects of the island and its topography.

We represent the topography as a simple continental slope "skirt" around the island in which the depth of the ocean linearly varies from zero at the island to the full (and constant) ocean depth at a distance $x_{T}$ both east and west of the island which we otherwise idealize as a thin linear barrier oriented north-south as shown in Figure 1.

In addition to providing a possibly more realistic representation of the island topography, the presence of the skirt, with its steep slope, also introduces fundamental changes in the dynamics. The ambient potential vorticity is $f / h$ where $f$ is the latitudinally varying Coriolis parameter and $h$ is the depth of the ocean, here represented as a fluid with a single constant density $\rho$. When the depth change is strong enough, the isolines of potential vorticity will tend to wrap around the island and close on themselves. This distinguishes the present study from other investigations of the role of coastal topography; e.g., Salmon (1992) for which the potential vorticity contours intersect the boundary and remain blocked rather than closed on themselves. When this closure happens, a free geostrophic mode is possible in which the motion can freely circulate along the closed potential vorticity contours and the nature of the circulation alters dramatically and the Island Rule must be modified substantially as will be shown below.

One of the interesting predictions of the Island Rule in models without island topography is the appearance of a recirculation region east of the island if the island extends sufficiently in the meridional direction to straddle both eastward and westward flows in the 


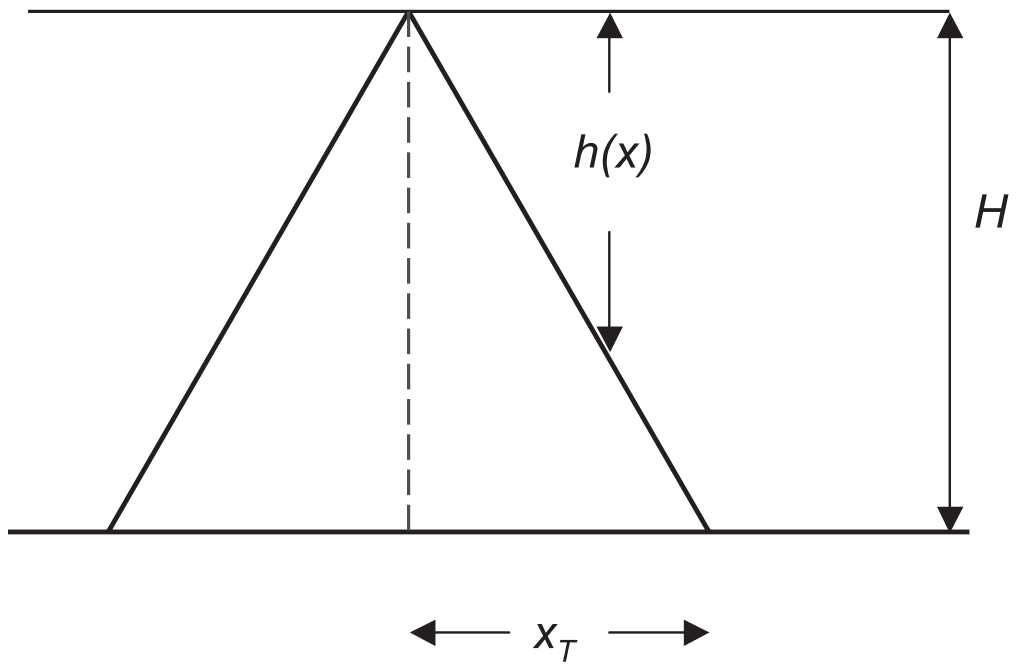

Figure 1. A schematic showing the east-west cross section of the island topography. The depth of the ocean is $h$ and beyond the island's skirt it is $H$. The half width of the skirt is $x_{T}$. The dashed line shows the island if no skirt is present.

Sverdrup interior (PPSH). The trapped region of fluid is a direct consequence of the Island Rule and, aside from a small leakage of fluid, represents a reservoir of fluid trapped to the island within the recirculation region. The alteration of the potential vorticity structure around the island as a consequence of the sloping topography will alter the circulation and in particular, the geometry and extent of the trapped fluid.

Section 2 discusses our fundamental model and presents the circulation integral that would determine the circulation around the island, at least in the linear limit, in the absence of topography. This will identify the proximate cause for the change in the Island Rule. Section 3 gives an introduction to our numerical results in the linear and moderately nonlinear regime and demonstrates a strong functional relation between the transport stream function and the potential vorticity over the region of the skirt. Section 4 describes a series of approximate analytical results that to a large measure clarify and explain key features of the numerical calculations especially in the linear regime. In Section 5 we present the results of strongly nonlinear calculations and the onset of time-dependent motion. Section 6 presents the results of laboratory experiments with the skirted island geometry. We sum up our results in Section 7 and describe the direction of future work.

\section{The model}

We consider a circular ocean basin of radius $R$ (Fig. 2). This is done for convenience for comparison to our laboratory experiments. The island is a thin, elongated barrier of half-length $0.7 R$ centered symmetrically in the basin. Around the island a topographic skirt, Figure 2a, produces a linearly increasing depth of the fluid in the longitudinal 

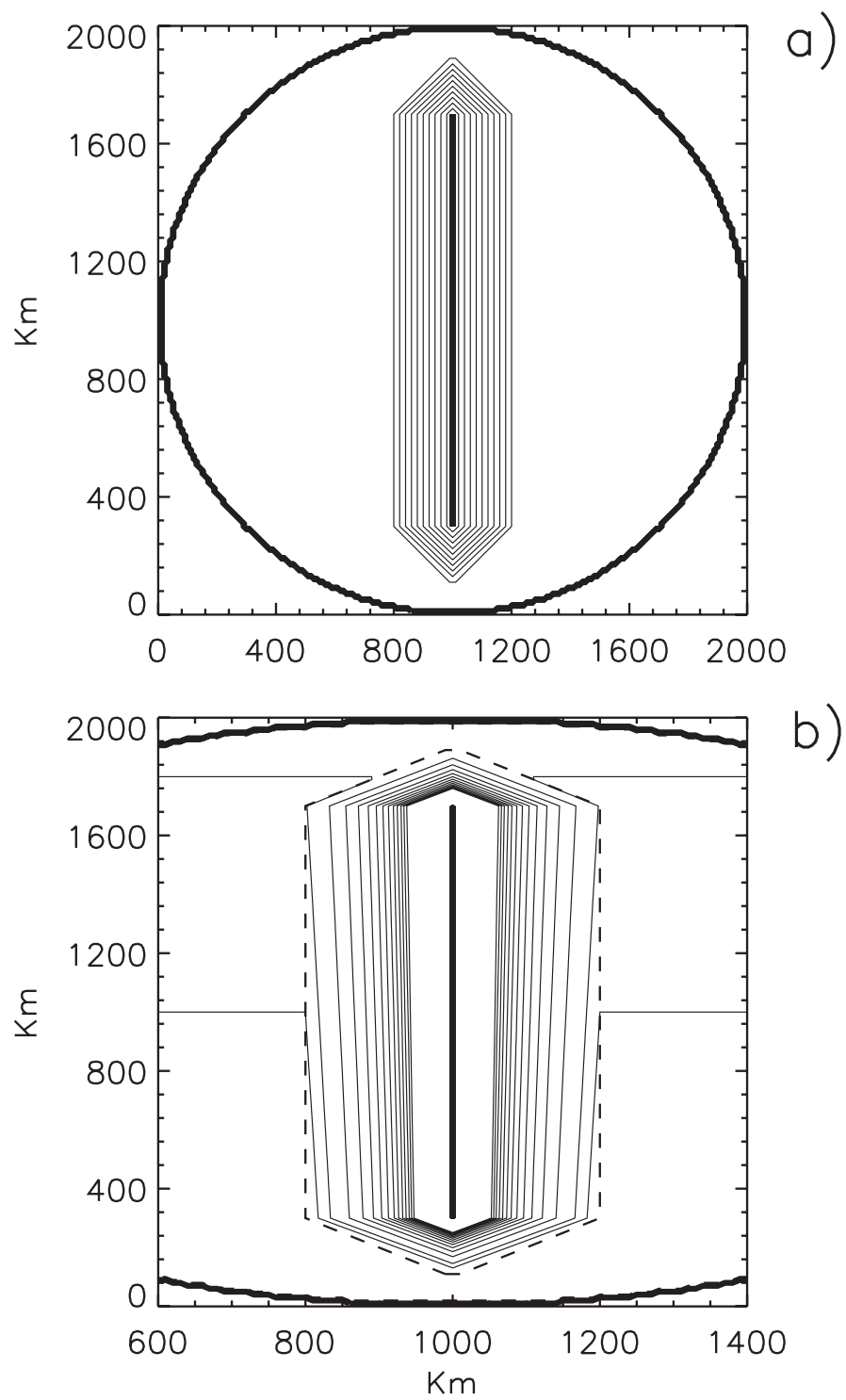

Figure 2. The island showing (a) the isobaths around the island and (b) the contours of ambient potential vorticity, $f / h$, showing the region of closed geostrophic contours.

direction from the island to a distance $x_{T}$ on each side of the island beyond which the fluid depth is a constant, $H$. Beyond the two tips of the central island the depth is linearly increased over the same distance to render the topography smooth and so to avoid abrupt changes in depth at the northern and southern tips of the islands. In our numerical experiments the basin has a radius of $1000 \mathrm{~km}$, and the width of the skirt, $x_{T}$, is $200 \mathrm{~km}$ 
although we have done some calculations with different values of $x_{T}$. The fluid depth decreases from zero at the island to a constant depth of 1,000 $\mathrm{m}$ at the edges of the skirt. The fluid is rotating with a Coriolis parameter, $f$, and is on the beta plane with a meridional gradient $\beta$. For the numerical calculations to be reported below we have chosen $f=10^{-4} \mathrm{sec}^{-1}$ at the central latitude of the basin and $\beta=1.25 \times 10^{-11} \mathrm{~m}^{-1} \mathrm{sec}^{-1}$. For these values the isolines of ambient potential vorticity, f/h, (Fig. 2b) are closed around the island over most of the skirt.

The fluid is driven in the numerical experiments by a wind stress of the same form as in PPSH, namely a stress directed in the azimuthal direction, linearly increasing with radius $r$ from the center of the basin,

$$
\vec{\tau}=\tau_{m}\left(\frac{r}{R}\right) \vec{\imath}_{\theta}
$$

where $\vec{\imath}_{\theta}$ is a unit vector in the azimuthal direction and $\vec{\tau}$ is the stress. This produces a constant wind stress curl $2 \tau_{m} / R$. For an anticyclonic wind stress $\tau_{m}$ is negative.

Our dynamical model is a forced, dissipative shallow water system whose governing equations can be written as

$$
\begin{gathered}
\frac{\partial \vec{u}}{\partial t}+(\zeta+f) \vec{k} \times \vec{u}=-\nabla B+\frac{\vec{\tau}}{\rho h}+D(\vec{u}), \\
\nabla \cdot(h \vec{u})=0,
\end{gathered}
$$

where

$$
B=\frac{1}{2}|\vec{u}|^{2}+p / \rho, \quad \zeta=\vec{k} \cdot \nabla \times \vec{u}
$$

and $\vec{k}$ is a vertical unit vector. The pressure is hydrostatic and can be related to a virtual free surface elevation, $\eta ; p / \rho=g\left(h+h_{b}+\eta\right)$, where $h_{b}$ is the height of the bottom above $z=-H$ where $H$ is the constant fluid depth off the topographic skirt. In fact, as evidenced by (2.2b), a rigid lid approximation is used throughout so that $h$ is the fluid thickness and is independent of time so that $\eta$ is related to the surface pressure simply as, $\eta=\frac{p_{s}}{\rho g}$. The dissipation term $D(\vec{u})$ is modeled as,

$$
D(\vec{u})=\frac{1}{h}(\nabla \cdot A h \nabla) \vec{u}-\frac{\lambda}{h} \vec{u}
$$

where the first term on the right-hand side of (2.4) represents lateral friction and the final term is a parameterization of bottom friction. We define parameters

$$
\delta_{M}=\left(\frac{A}{\beta}\right)^{1 / 3}, \quad \delta_{I}=\left(\frac{\tau_{m}}{\rho R \beta^{2} H}\right)^{1 / 2} \delta_{S}=\frac{\lambda}{\beta H}
$$


as definitions of the Munk layer thickness, the inertial boundary layer thickness and the Stommel boundary layer thickness as in PPSH although the variable topography renders these definitions rather formal. Nevertheless, we will typically use the ratios, $\delta_{I} / \delta_{M}$ and $\delta_{s} / \delta_{M}$, as measures of nonlinearity and bottom friction for comparison with the flat bottom case discussed in PPSH. In most of our numerical calculations the ratio $\delta_{s} / \delta_{M}$, has been chosen, as in PPSH, to be 0.678 so that our control parameter for nonlinearity will be $\delta_{I} / \delta_{M}$.

The use of the rigid lid approximation (2.2b) allows us to define a stream function,

$$
h \vec{u}=\vec{k} \times \nabla \psi .
$$

This allows (2.2a) to be rewritten

$$
q \nabla \psi=\nabla B-\frac{\vec{\tau}}{\rho h}-D(\vec{u})+(\vec{k} / h) \times \nabla \frac{\partial \psi}{\partial t}
$$

where $q$ is the potential vorticity

$$
q=\frac{f+\zeta}{h}, \quad f=f_{o}+\beta y .
$$

The form (2.7) will be found particularly useful for steady flows. Boundary conditions are:

$$
\begin{aligned}
& \psi=0, \quad r=R, \\
& \psi=\Psi_{I}, \quad \text { on the island. }
\end{aligned}
$$

The constant, $\Psi_{I}$, thus yields the total transport around the island from the western to eastern sub-basins. The island constant, $\Psi_{I}$, must be determined as part of the solution.

The Island Rule as obtained by Godfrey (1989) is most directly obtained by the integral of the tangential component of the momentum equation around the carefully chosen circuit in Figure 3. With only a very slight generalization of the procedure in PPSH to include variable topography, this leads to the result

$$
\begin{aligned}
\left(f_{n}-f_{s}\right) \Psi_{I}=-\oint_{C} \frac{\vec{\tau}}{\rho} \cdot d \vec{s}-\oint_{C} h D(\vec{u}) & \cdot d \vec{s}+\frac{\partial}{\partial t} \oint_{C} h \vec{u} \cdot d \vec{s} \\
& -\int_{x_{I}}^{x_{e}} \frac{\partial}{\partial y}\left\{[u v h]_{y_{n}}-[u v h]_{s}\right\} d x-\oint_{C} p \frac{\partial h}{\partial s} d s
\end{aligned}
$$

where $\Psi_{I}$ is the value of the stream function on the island (centered at $x=0$ ) and $f_{n}$ and $f_{s}$ are the values of the Coriolis parameter at the northern and southern edges of the island. We define the Coriolis parameter as $f=f_{o}+\beta y$ where $y=0$ is at the mid-latitude of the basin. Note that the topography extends northward to $y_{N}=y_{n}+\Delta_{T}$ and southward to $y_{S}=y_{s}-\Delta_{T}$. In the absence of dissipation, time dependence, nonlinear fluxes of 
a)

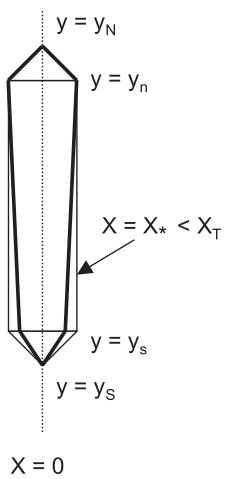

b)

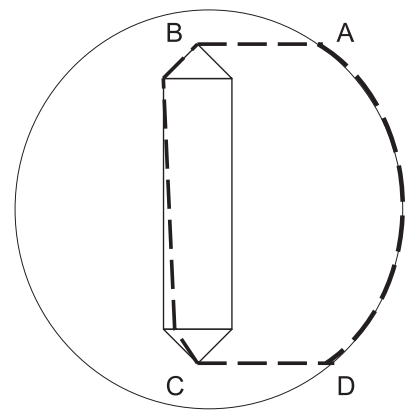

c)

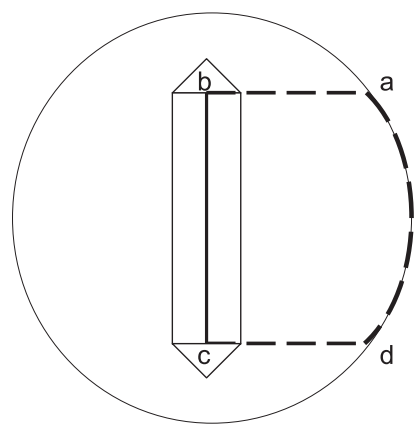

Figure 3. The contours for the Island Rule integral. (a) The contour (heavy solid line) around a isoline of of potential vorticity. (b) the contour (ABCDA) for the determination of $\psi_{T}$. (c) for the island rule integral in (2.10).

momentum and bottom relief, the value of $\Psi_{I}$ is completely determined by the integral of the wind stress on the circuit of Figure 3c. The presence of dissipation can be ignored on the chosen circuit if, as is the case for a flat bottomed ocean, the dissipation on the western edge of the island is negligible. The dissipation is normally negligible except in a western boundary current on the eastern side of the island. The new term when there is bottom relief is the last term which is the integral of the pressure force on the sloping topography when integrated along the contour in the region where the depth is diminishing on the flanks of the island. We shall see that this term is generally important when the island has a skirt of the kind we are considering but more importantly, the presence of the topography so alters the circulation by altering the potential vorticity distribution that the idea that the dissipation can be ignored to the west of the island becomes problematic.

\section{Steady solutions: numerical results}

We first investigate the nature of the flow and the changes wrought by the presence of the topographic skirt with calculations made with the barotropic version of the Princeton Ocean Model (POM); (Blumberg and Mellor, 1987, Mellor, 1992). Solutions to (2.2) were found by discretizing the equations on a square grid with a $10-\mathrm{km}$ spacing. The principal control parameter is the ratio $\delta_{I} / \delta_{M}$ which is a measure of the nonlinearity in the boundary layers. All the geometrical parameters are fixed as described above and the variation of the control parameter is accomplished by altering $\delta_{I}$ by increasing the magnitude of the wind stress $\tau_{m}$.

Figure 4 shows the result of the anticyclonic, wind forced circulation for the case of a flat bottomed ocean; i.e., an island with no skirt and is presented for comparison with the calculation of PPSH. The circulation is qualitatively identical to the calculation reported in 


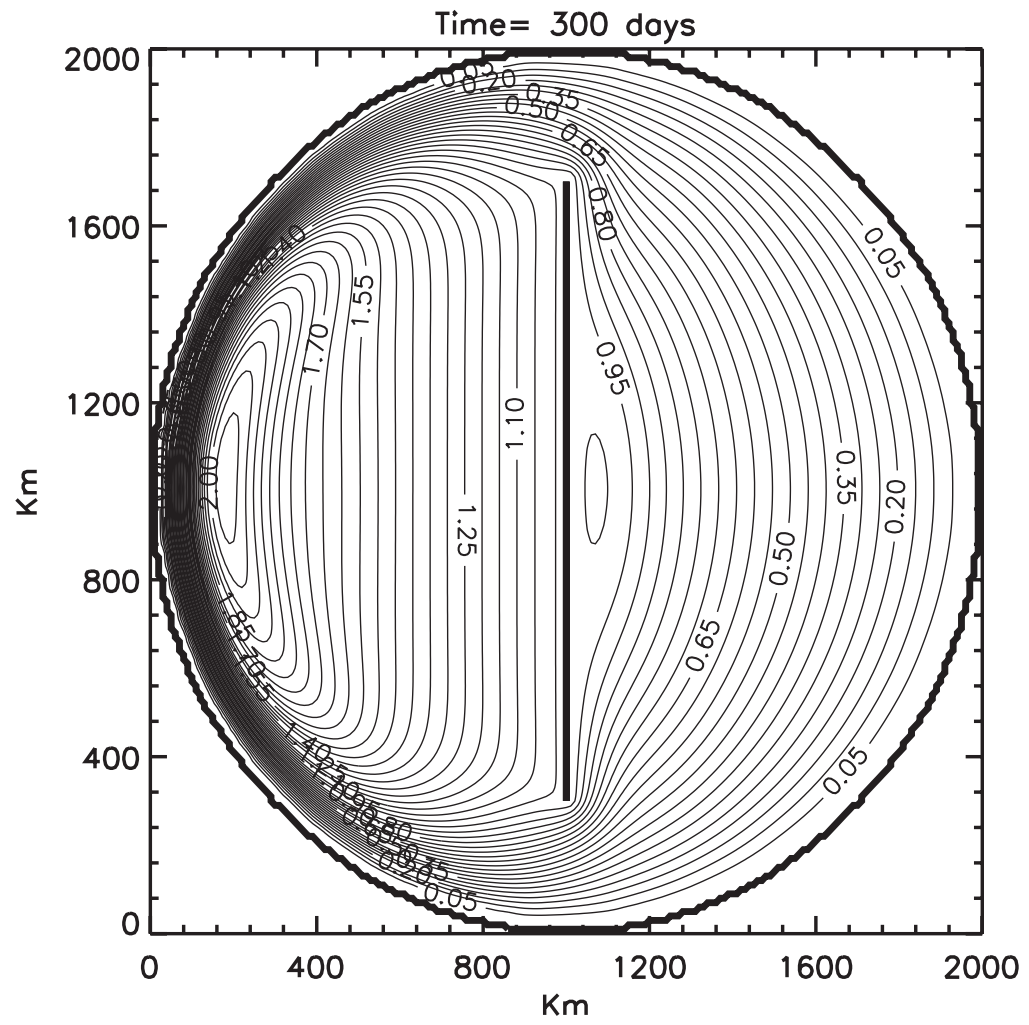

Figure 4. The circulation for the island with no skirt (flat bottomed ocean) for $\delta_{I} / \delta_{M}=0.25$.

PPSH (where the island was slightly longer) for the same parameter setting $\delta_{I} / \delta_{M}=0.25$, essentially a linear setting. The circulation shows the appearance of the recirculation region east of the island. The stagnation points on the island fix the north-south extent of the recirculation and these points are determined by the condition that the stream function on the island $\Psi_{I}$ is equal to the Sverdrup stream function at that latitude and longitude (see PPSH for details). Since $\Psi_{I}$ is determined by the Island Rule (2.10) we can anticipate changes in the recirculation if the Island Rule no longer applies. Indeed, for the same parameter setting but with the presence of the topographic skirt, the solution becomes radically different and is shown in Figure 5. The most obvious change is that there is a closed, tight circulation around the island on its topographic skirt that, to a very good approximation, follows the closed ambient potential vorticity contours shown in Figure 2b. The bottom topography is so strong that the local dynamics is dominated by the topographic control of the circulation. At the same time, the eastern recirculation region of the flat bottom case has disappeared although the slight bowing of the Sverdrup circulation is still visible to the east of the island. In addition, the circulation to the west of the island is nearly as strong as to the east and the dissipation is roughly of the same order on each side 


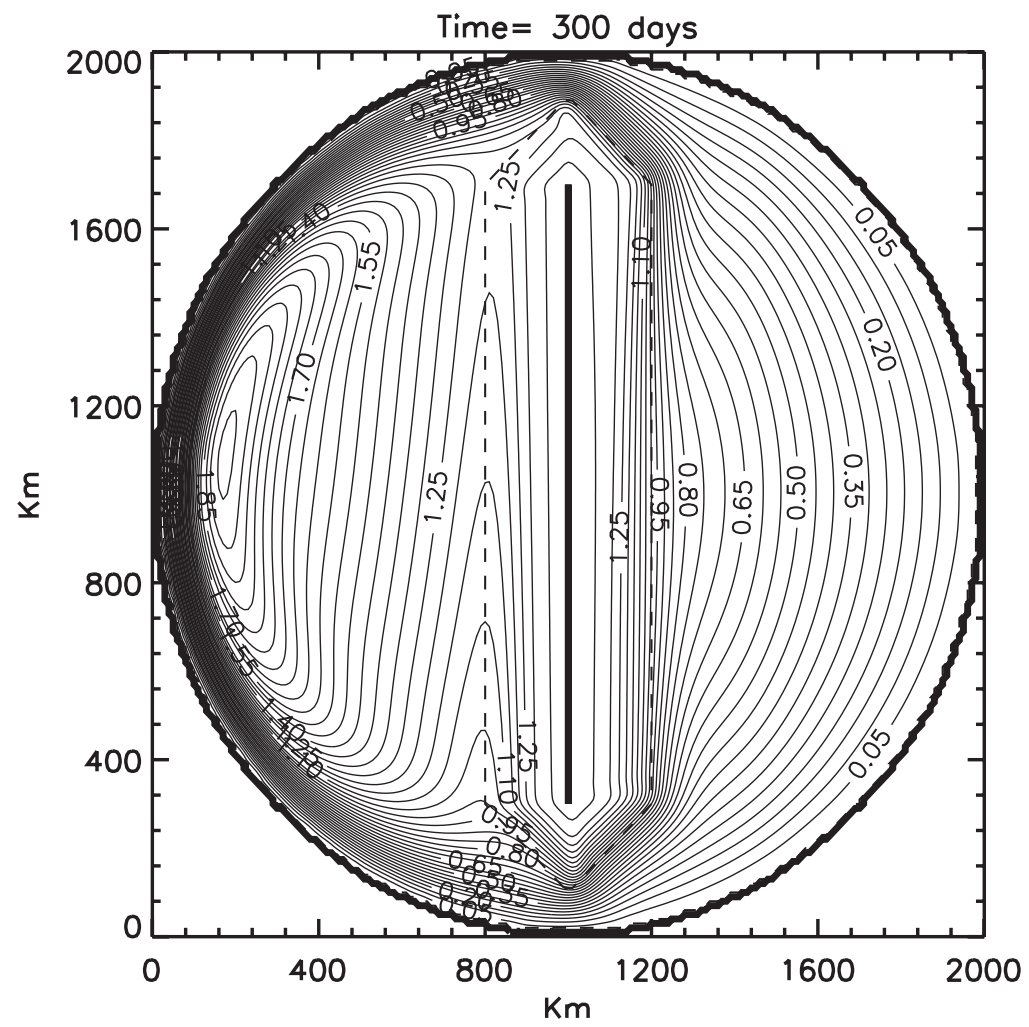

Figure 5. As in Figure 4 except that the island now has a skirt topography whose boundary is indicated by the dashed contour.

of the island. This implies that dissipation cannot be ignored to the west of the island as is assumed in the usual Island Rule derivation.

A dramatic change of direction of the streamlines on the western edge of the skirt is observed as a northward flow to the west of the island suddenly turns in an inverted $\mathrm{V}$-shaped meander to the southwest. Note that this is an essentially linear regime, so a change in the wind stress from anticyclonic to cyclonic would change the direction of the flow but will leave the pattern of the flow unchanged. This is confirmed in a cyclonically forced calculation of the same strength but which we do not show for the sake of brevity. As well as altering the pattern of the flow, the total transport between the western and eastern sub basins; i.e., the transport around the island is altered significantly. For the flow of Figure 4 (no skirt) that transport, $\Psi_{I}$, is 0.98 Sverdrups while with the skirt, Figure 5, $\Psi_{I}$ is 1.36 Sverdrups, about $38 \%$ increase.

The control of the flow over the skirt by the topography is made manifest by a strong relation between the stream function and the potential vorticity in that region. Figure 6 shows a scatter plot of potential vorticity, $q$, versus $\psi$ and a clear functional relation is 

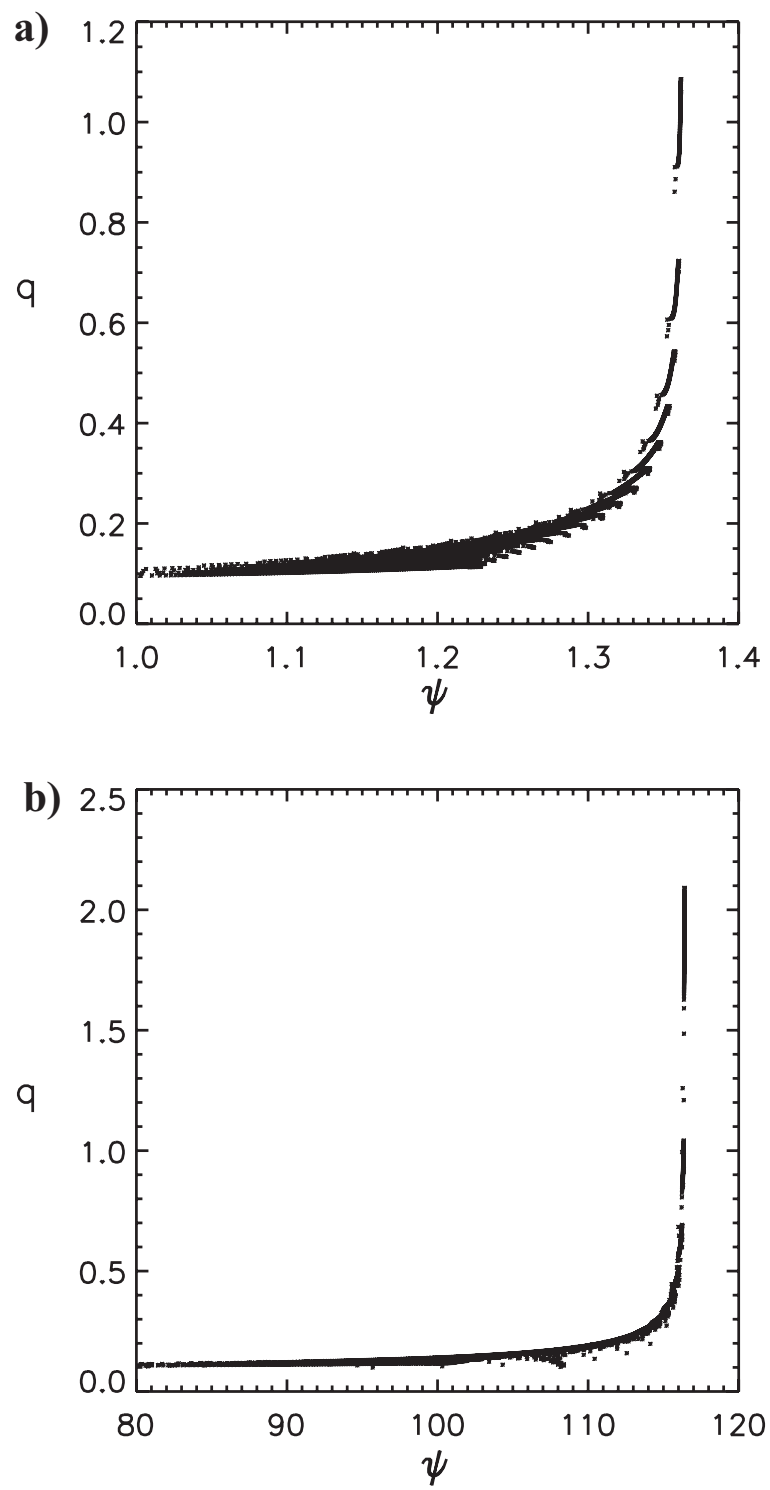

Figure 6. Scatter plots of $q$ vs. $\psi$ on the western skirt. (a) The linear case $\delta_{I} / \delta_{M}=0.25$. (b) a nonlinear case, $\delta_{I} / \delta_{M}=2$. In each case the forcing is anticyclonic.

observed. In panel (a) of the figure, for the linear solution, $\delta_{I} / \delta_{M}=0.25$, the scatter plot is a bit fuzzy but as the inertial terms begin to dominate for larger nonlinearity the relation becomes tighter as shown in panel (b) of the figure. A detailed analysis, not shown here, reveals that for depths smaller than about 700 meters, the bottom drag, including the bottom pressure torque nearly balances the wind forcing locally and the lateral friction 


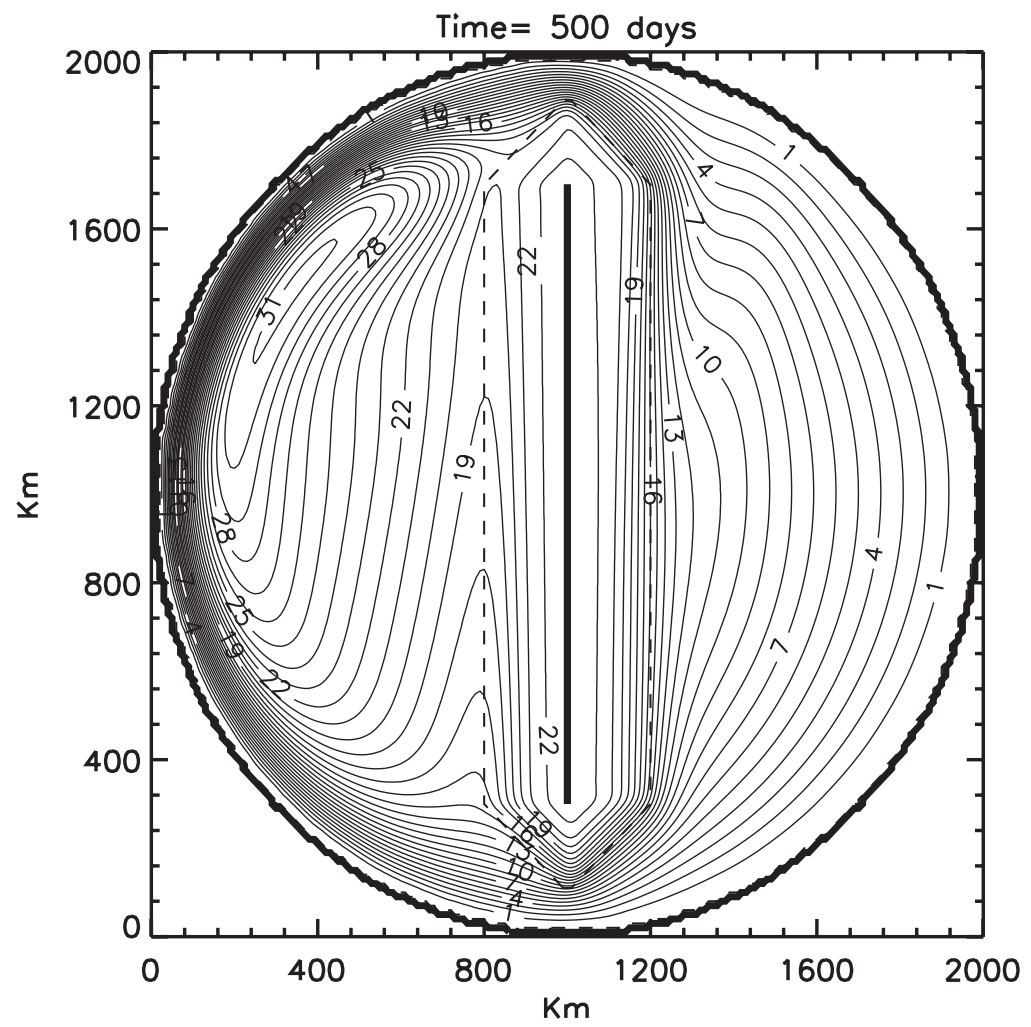

Figure 7. As in Figure 5 except that $\delta_{I} / \delta_{M}=1$.

term is small. The flow is nearly along contours of $f / h$, the ambient potential vorticity. We shall exploit these diagnostic relations in our analytic discussion below.

As inertia becomes more important, the advection of relative vorticity by the flow field becomes more evident and the north-south symmetry of the flow is clearly broken. Figure 7 shows the flow for $\delta_{I} / \delta_{M}=1$. The center of the gyre in the western sub basin is moved northward by the anticyclonic flow and a weak meander appears northwest of the island and a nascent meander is seen east of the island in the northeastern quadrant. As the forcing is increased further and the flow becomes more nonlinear, the normally expected symmetry (see Kiss, 2002 for example) between cyclonic and anticyclonic forcing, where one is a mirror image of the other around the central latitude is lost, as shown in Figure 8 for $\delta_{I} / \delta_{M}=2$. In panel (a) the anticyclonic circulation has developed an intense, deep meander to the east of the island produced by streamlines adjacent to the skirt that are constrained to move directly southward generating positive relative vorticity. It is interesting to note that a similar pair of oppositely signed vortices is produced also in the absence of the skirt topography as was found in PPSH.

However, with cyclonic forcing, as shown in panel (b) the meander to the east of the 

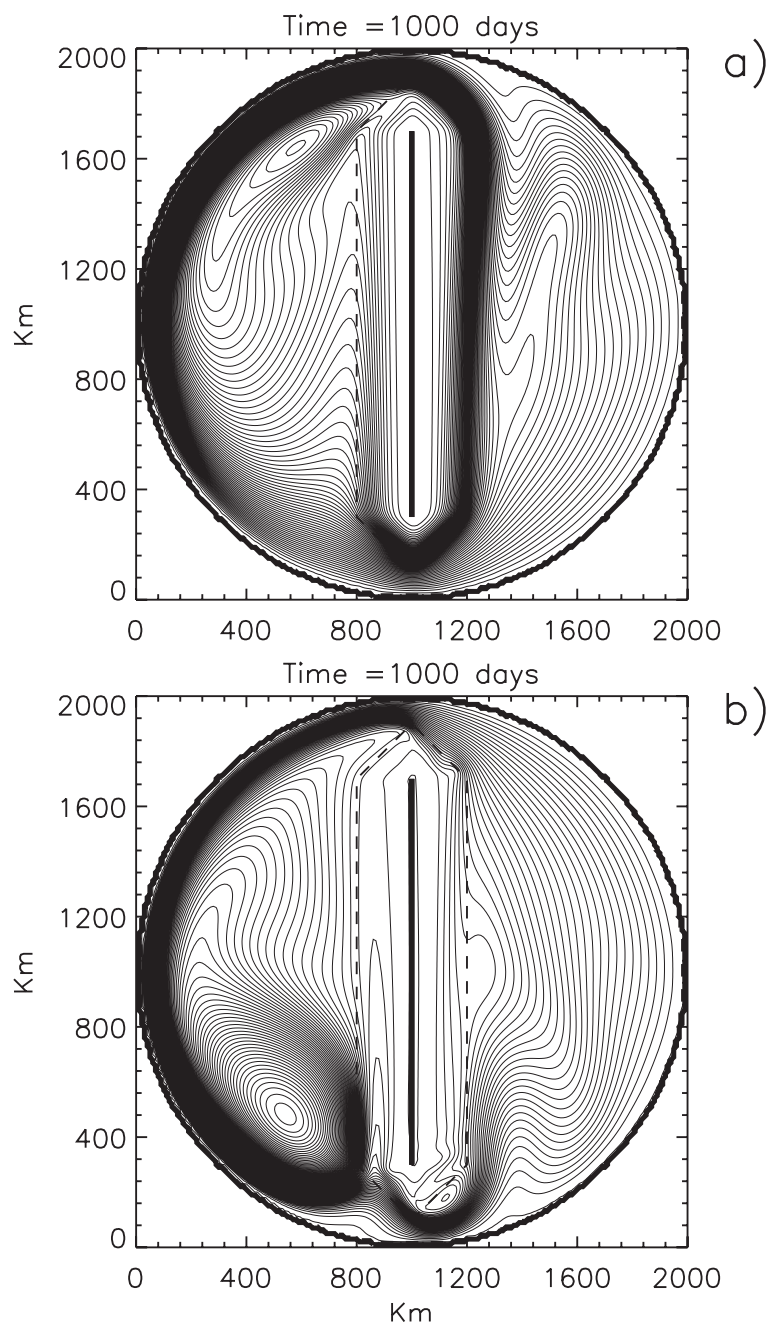

Figure 8. The circulation for $\delta_{I} / \delta_{M}=2$. (a) Anticyclonic forcing. (b) Cyclonic forcing.

island in the southeastern quadrant is very much weaker and the recirculation to the west of the island in the southwestern quadrant is significantly more symmetric than its counterpart in the northwestern quadrant with anticyclonic forcing. This break in the north-south mirror symmetry under reversal of the sign of the wind stress is due to the presence of the imposed potential vorticity gradient in the $x$ direction due to the topography of the skirt. We shall generally limit our discussion to the anticyclonic case but this loss of symmetry must be kept in mind. For larger values of the ratio $\delta_{I} / \delta_{M}$, around 2.1, we find that the circulation becomes time dependent in the anticyclonic case probably due to an instability in the boundary layers on the western boundary of the basin and/or in the strong standing 


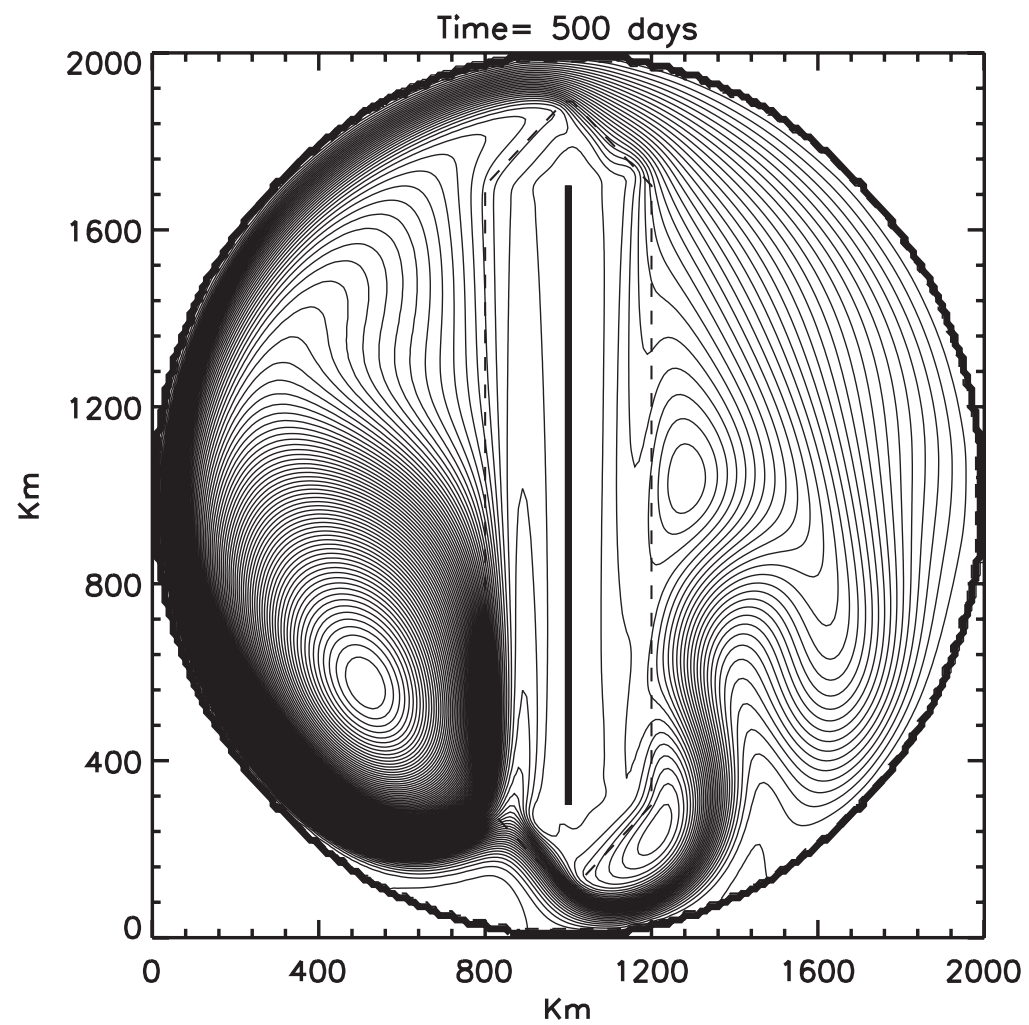

Figure 9. The circulation for $\delta_{I} / \delta_{M}=2.5$ for cyclonic forcing.

wave field in the northeast quadrant. We emphasize that in the absence of the topographic skirt we have found complete symmetry between cyclonic and anticyclonic forcing in agreement with earlier studies, e.g. PPSH and Kiss (2002).

In this nonlinear regime, when the forcing is cyclonic the steady solution is retained for larger values of $\delta_{I} / \delta_{M}$ for which the anticyclonic forcing yields time-dependent solutions. Figure 9 shows the steady solution for cyclonic forcing when $\delta_{I} / \delta_{M}=2.5$ when the anticyclonic forcing yields a time-dependent solution to be discussed in detail in Section 5. This asymmetry between anticyclonic and cyclonic forcing is related to the direction of propagation of topographic Rossby waves on the skirt which tend to propagate their phase clockwise around the island. A topographic wave can then be held stationary by a cyclonic steady circulation. Depending on the value of the velocity the stationary wave can have a strongly meandering pattern. The nature of this asymmetry has been previously discussed by LaCasce et al. (2008) for the case of free circulations but the same considerations apply to our forced case. In the circulation shown in Figure 9 two strong recirculation eddies are held in place on the eastern side of the island's skirt while a very powerful meander occupies a substantial part of the southeastern quadrant of the basin. The strong recircula- 
tion in the southwestern basin quadrant is a familiar consequence of the excess vorticity advected southward by the western boundary current. In Section 5 we will return to the discussion of the strongly time-dependent circulations when the flow is anticyclonic, but we point out that that configuration would be expected to be stable if only the positive sign of $d q / d \psi$ on the skirt is considered. We suppose, therefore, that the anticyclonic circulation is more likely rendered unstable by details of the viscous boundary layer structure on the basin boundary.

\section{Linear theory}

\section{a. The recirculation}

We present in this section an approximate analytical theory for key elements of the steady circulation in the linear limit that give insight into the fundamental nature of the role of topography. Starting from (2.7) and limiting attention to steady flows,

$$
q \nabla \psi=\nabla B-\frac{\vec{\tau}}{\rho h}-D(\vec{u})
$$

where, over the region of the skirt, excluding the tip regions (which we will discuss below),

$$
\begin{aligned}
h & =h_{x}|x|, & & |x| \leq x_{T}, \\
& =H & & |x| \geq x_{T}
\end{aligned}
$$

so that $h_{x}=H / x_{T}$. The numerical results of Section 3 suggest that over the region of the skirt where the $q$ isolines are closed, the potential vorticity is functionally related to the stream function and in this section we will take that as a central hypothesis and attempt to determine that relation as well as the value of $\Psi_{I}$. So we assume

$$
\psi=\psi(q)
$$

over most of the skirt, so that (4.1) becomes,

$$
q \nabla q \frac{d \psi}{d q}=\nabla B-\frac{\vec{\tau}}{\rho h}-D(\vec{u})
$$

We now integrate (4.4) on a closed circuit in Figure 3a that corresponds to a contour of constant $q$. In the linear limit, i.e. when $\zeta \ll f$, those contours correspond to the contours of constant ambient potential vorticity of Figure $2 \mathrm{~b}$. The topographic variation dominates the variation of $q$ since $\beta\left(y_{n}-y_{s}\right) / f<1$ and those contours will be nearly coincident with the bathymetry as shown in Figure $2 \mathrm{~b}$. However the departure of the $q$ contours from the isobaths, while small, is not completely negligible and so we will include the variation of the Coriolis parameter in our calculation. Assuming the above, the integral of the first and second terms of (4.4) vanish on a closed curve of constant ambient potential vorticity yielding, 


$$
\oint_{C_{q}} \frac{\vec{\tau}}{\rho h} \cdot d \vec{s}+\oint_{C_{q}} D(\vec{u}) \cdot d \vec{s}=0
$$

Naturally, (4.5) follows directly from (4.1) with no assumption that $\psi$ is a function of $q$ as long as the contour is a closed curve on which $q$ is constant. The further identification of the contour with a streamline is exploited below to find an explicit representation of the stream function field.

Note that (4.5) implies that the bottom pressure torque, important locally, integrates to zero on a closed streamline coincident with the contours of $q$. Were we able to find an analytic solution for the boundary layer structure over the skirt in the linear limit, we could evaluate the second term in (4.5) explicitly. In the joint presence of $\beta$ and the topography this is difficult. Instead we content ourselves for the sake of analytical simplicity with representing the friction as purely bottom drag,

$$
D(\vec{u})=-\frac{\lambda}{h} \vec{u}=-\lambda \vec{k} \times \nabla \psi / h^{2}
$$

so that,

$$
\begin{aligned}
\oint_{C_{q}} \frac{\vec{\tau}}{\rho h} \cdot d \vec{s} & =\lambda \oint_{C_{q}} \frac{1}{h^{2}} \nabla \psi \cdot \vec{n} d s \\
& =\lambda \frac{d \psi}{d q} \oint_{C_{q}} \nabla q \cdot \vec{n} / h^{2} d s
\end{aligned}
$$

where $\vec{n}$ is the unit vector normal to the contour. The balance in (4.7) between the wind stress and the bottom friction is also suggested by our numerical results as described in the previous section.

Both the wind stress and the ambient potential vorticity are symmetric about the island on the skirt so the integrals in (4.7) can each be approximated as integrals along only the right-hand side of the island in Figure 3a.

Consider first the integral of the stress tangent to the island on the nearly meridional line segment of constant $q$ in the interval $\left(y_{s}, y_{n}\right)$ on the eastern side of the island. The isoline of $q$ has an outward normal

$$
\hat{n}=\frac{\hat{i}-\frac{\beta}{h_{x} q} \hat{j}}{\left\{1+\frac{\beta^{2}}{h_{x}^{2} q^{2}}\right\}^{1 / 2}}
$$

and a tangent to the contour $\hat{t}=\hat{k} \times \hat{n}$ where $\hat{k}$ is the vertical unit vector, since 


$$
\vec{\tau}=\frac{\tau_{m}}{R} r[\hat{j} \cos \theta-\hat{i} \sin \theta]
$$

The integral of the stress (4.7) on this line segment, since $d s=d y\left\{1+\frac{\beta^{2}}{h_{x}^{2} q^{2}}\right\}^{1 / 2}$ yields,

$$
\begin{aligned}
\int_{y_{s}}^{y_{n}} \frac{\tau_{m}}{\rho R}\left[\frac{x}{h}-\frac{y \beta}{h_{x} q h}\right] d y & =\int_{y_{s}}^{y_{n}} \frac{\tau_{m}}{\rho R h_{x}}\left[1-\frac{\beta y}{f_{o}+\beta y}\right] d y \\
& =\frac{\tau_{m}}{\rho R} \frac{f_{o}}{\beta h_{x}} \ln \left\{\frac{1+\beta y_{n} / f_{o}}{1+\beta y_{s} / f_{o}}\right\} \\
& \approx \frac{\tau_{m}}{\rho R h_{x}}\left(y_{n}-y_{s}\right)
\end{aligned}
$$

and where $f_{o}$ is the Coriolis parameter at $y=0$, the mid-latitude of the basin. The last step in (4.10) assumes $\beta\left(y_{n}-y_{s}\right) \ll f$, an approximation that, generally, will not be applied. The segment on the western side of the island yields the same result so that the total contribution of the stress from the long sides of the $q$ contours is double the result in (4.10).

For the triangular extensions of the island and the $q$ contours, the variation of $f$ is negligible and we can identify the isolines of $q$ with the isobaths for this region. The outward normal of the contour is then just $\hat{n}=(\hat{i}+\hat{j}) / 2^{1 / 2}$ and the stress integral on this small segment from $\left(x_{*}, y_{n}\right)$ to $\left(0, y_{n}+x_{*}\right)$ where $x_{*}$ is the position of the $q$ contour at $y_{n}$ is just

$$
\begin{aligned}
\int \frac{\vec{\tau}}{\rho h} \cdot \hat{t} d s & =\frac{\tau_{m} q}{\rho R f_{n}}\left[x_{*}\left(y_{n}+x_{*}\right)\right] \\
& =\frac{\tau_{m}}{\rho R h_{x}}\left\{y_{n}+\frac{f_{n}}{h_{x} q}\right\}
\end{aligned}
$$

and, again, because of the east west symmetry of the $q$ isolines the western side of the island contributes a term equal to (4.11). A similar calculation on the southern extension tips of the island yield a similar term. With proper attention to sign the total stress integral becomes, in our approximation,

$$
\oint_{C} \frac{\vec{\tau} \cdot \hat{t}}{\rho h} d s=\frac{2 \tau_{m}}{\rho R h_{x}}\left\{\frac{f_{o}}{\beta} \ln \left(\frac{f_{n}}{f_{s}}\right)+\left(y_{n}-y_{s}\right)+\frac{f_{n}+f_{s}}{q h_{x}}\right\}
$$

and $f_{n}$ and $f_{s}$ are the Coriolis parameters evaluated at $y_{n}$ and $y_{s}$, respectively. It is important to note that the contribution of the stress to the integral from the narrow segments at the tips of the island is as large as the contribution from the long segments east and west of the island. The smallness of the stress on the latter segments compensates for the greater length of the segment. 
A similar integration of the dissipation term, always keeping in mind that $q$ is constant on the contour in (4.7), yields for that integral, after a considerable amount of algebra,

$\lambda \frac{d \psi}{d q} \oint_{C_{q}} \nabla q \cdot \vec{n} / h d s=-2 \lambda \frac{d \psi}{d q} h_{x}\left[\frac{q^{4}}{2}\left\{1+\frac{\beta^{2}}{q^{2} h_{x}^{2}}\right\} \frac{\left(f_{n}+f_{s}\right)\left(y_{n}-y_{s}\right)}{\left(f_{n} f_{s}\right)^{2}}+2 \frac{q^{3}\left(f_{n}^{2}+f_{s}^{2}\right)}{h_{x}\left(f_{n} f_{s}\right)^{2}}\right]$

so that we finally obtain from (4.7)

$$
\frac{d \psi}{d q}=-\frac{2}{\lambda} \frac{\tau_{m}}{\rho R h_{x}^{2}} \frac{\left[\frac{f_{o}}{\beta} \ln \left(\frac{f_{n}}{f_{s}}\right)+\left(y_{n}-y_{s}\right)+\frac{f_{n}+f_{s}}{q h_{x}}\right]}{\left[q^{4}\left\{1+\frac{\beta^{2}}{q^{2} h_{x}^{2}}\right\} \frac{\left(f_{n}+f_{s}\right)}{\left(f_{n} f_{s}\right)^{2}}\left(y_{n}-y_{s}\right)+4 \frac{q^{3}\left(f_{n}^{2}+f_{s}^{2}\right)}{h_{x}\left(f_{n} f_{s}\right)^{2}}\right]} .
$$

The term $\frac{\beta^{2}}{q^{2} h_{x}^{2}}=\frac{\beta^{2}}{f^{2}} x^{2} \leq \frac{\beta^{2}}{f^{2}} x_{T}^{2}$ which for our parameters is $O\left(5 \times 10^{-4}\right)$ and is negligible in (4.14). Carrying out the integral in (4.14) and using the condition that $\psi=\Psi_{I}$ on $h=0$, i.e. at the island, where $q$ becomes infinite, we obtain, using the notation:

$$
\begin{aligned}
a & =\frac{f_{o}}{\beta} \ln \left(\frac{f_{n}}{f_{s}}\right)+\left(y_{n}-y_{s}\right), \\
b & =\frac{f_{n}+f_{s}}{h_{x}}, \\
c & =\frac{f_{n}+f_{s}}{\left(f_{n} f_{s}\right)^{2}}\left(y_{n}-y_{s}\right) \\
d & =\frac{4\left(f_{n}^{2}+f_{s}^{2}\right)}{h_{x}\left(f_{n} f_{s}\right)^{2}} \\
\psi-\Psi_{I}=\frac{-2 \tau_{m}}{\rho R h_{x}^{2} \lambda}\left[-\frac{b}{3 d q^{3}}\right. & \left.+(a d-b c)\left\{\frac{c^{2}}{d^{4}} \ln \left(\frac{q}{q+d / c}\right)+\frac{c}{d^{3} q}-\frac{1}{2 d^{2} q^{2}}\right)\right]
\end{aligned}
$$

If, in addition, we were to assume that $\beta\left(y_{n}-y_{s}\right) / f_{o} \ll 1$ then the dominant term in (4.14) is simply

$$
\begin{aligned}
\frac{d \psi}{d q} & =-2 \frac{f_{o}^{3}}{\lambda q^{4}} \frac{\tau_{m}}{\rho R h_{x}^{2}} \\
\rightarrow \psi-\Psi_{I} & =\frac{2}{3} \frac{f_{o}^{3}}{\lambda q^{3}} \frac{\tau_{m}}{\rho R h_{x}^{2}}
\end{aligned}
$$

for the wind stress given by (2.1). Figure 10 shows the comparison of the full integral (4.16) and the approximation (4.17b). In each case the $\psi-\Psi_{I}$ is scaled by $-2 \frac{\tau_{m}}{\rho \lambda R h_{x}^{2}}$. It's clear that the simple approximation (4.17) does a surprisingly good job qualitatively representing the variation of $\psi$ on the skirt although at the outer closed contour, where 


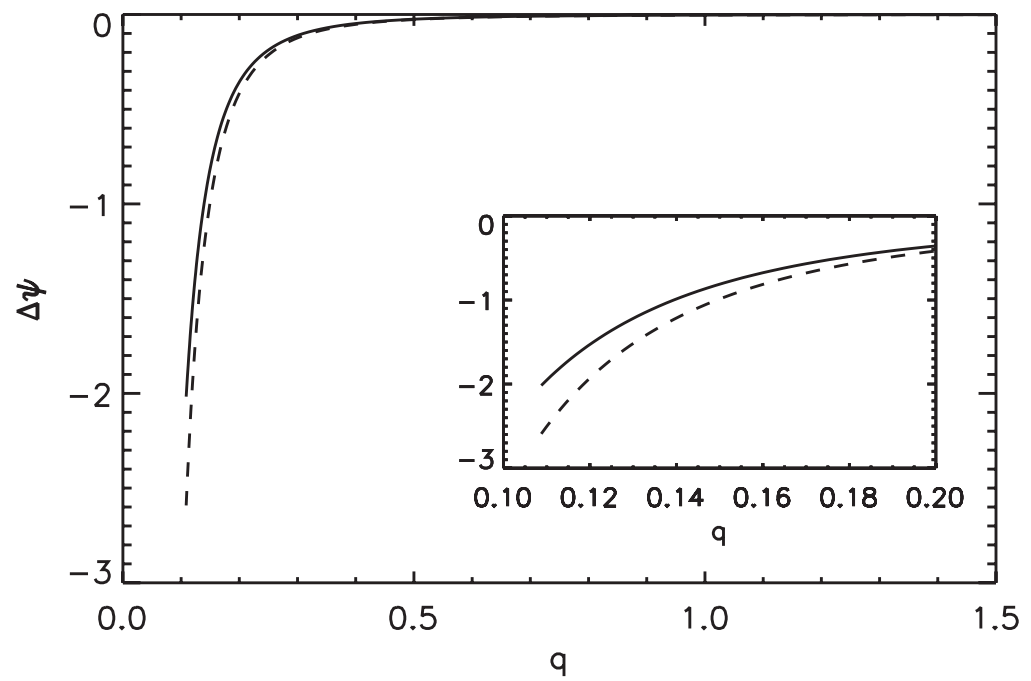

Figure 10. The square bracket in (4.16) (solid) and the approximation (4.17) (dashed), over the full range of $q$. In the insert the variation near the outermost closed pv contour.

$q=\frac{f_{n}}{H}$ (i.e. the left-hand edge of Fig. 10-insert) there is a discrepancy of about $20 \%$ between (4.16) and (4.17) with the latter an overestimate.

The comparison of the linear theory with the numerical experiment is shown in Figure 11. The form agrees remarkably well if the island constant $\Psi_{I}$ is evaluated from the numerical solution. We consider the agreement very satisfactory considering the approximations we have used.

To obtain an analytical expression for $\Psi_{I}$ we reconsider the integral as in (2.10) but we move the contour in Figure $3 \mathrm{c}$ to that shown in Figure $3 \mathrm{~b}$ so that now it lies just beyond the region of the skirt on the north and south of the island and closes west of the island along the $q$ contour that starts at $\left(-x_{T}, y_{n}\right)$ and runs southeastward along this contour until it enters the triangular region at $\left(-x_{T}+d, y_{s}\right)$ where

$$
d=x_{T} \frac{\beta\left(y_{n}-y_{s}\right)}{f_{n}} .
$$

The contour then coincides with the outermost closed contour on this segment west of the island.

Note that this opens up a thin wedge on the western edge topography in which the $q$ contours are not closed but traverse the skirt from southeast to northwest.

On that contour in Figure 3b,

$$
-\oint_{C_{o}} f \frac{\nabla \psi}{H} \cdot d \vec{s}=\oint_{C_{o}} \frac{\vec{\tau}}{h} \cdot d \vec{s}-\lambda \oint_{C_{o}} \frac{\nabla \psi}{h^{2}} \cdot \vec{n} d s
$$




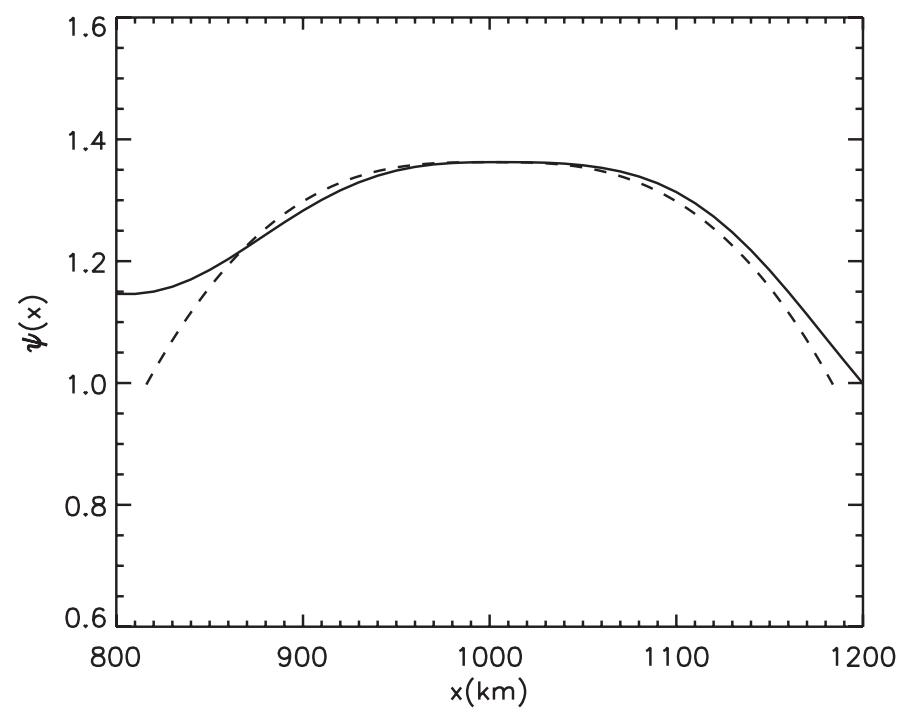

Figure 11. The variation of $\psi$ over the skirt at $y=0$. Solid line: from the numerical calculation of

Figure 5. Dashed line: using (4.16) and matching $\psi_{I}$ to the numerical solution.

On the western segment of the contour, where $q=f_{n} / H$ the stream function is the constant

$$
\psi=\psi\left(-x_{T}, y_{n}\right) \equiv \psi_{T}
$$

as well as on the eastern segment of the contour that coincides with the eastern boundary of the basin where it is zero. Thus the term on the left-hand side of (4.19) can be shown to be

$$
-\oint_{C_{o}} f \frac{\nabla \psi}{H} \cdot d \vec{s}=-\frac{\left(f_{N}-f_{S}\right)}{H} \psi_{T}
$$

where $f_{N}=f\left(y_{n}+x_{T}\right), f_{S}=f\left(y_{s}-x_{T}\right)$, i.e. the Coriolis parameters at the northern and southern tips of the skirt e.g. at $y_{N}=y_{n}+x_{T}, y_{S}=y_{s}-x_{T}$. Note that $h=H$ on this part of the contour. On the part of the contour that follows the western segment of the $q$ isoline the wind stress contribution balances the dissipation; this is a consequence of (4.13). Thus the remaining terms in (4.19), ignoring the dissipation in the region east of the island can be shown to yield (see PPSH), for $\psi_{T}$

$$
\begin{aligned}
\psi_{T} & =\frac{-\tau_{m}}{\rho \beta R}\left[x_{e}\left(y_{N}\right)+2 \frac{R^{2}}{\left(y_{N}-y_{S}\right)} \sin ^{-1}\left(y_{N} / R\right)\right], \\
x_{e}\left(y_{N}\right) & =\left[R^{2}-y_{N}^{2}\right]^{1 / 2}
\end{aligned}
$$

which, we note, is independent of $\lambda$. If we combine (4.22) with the approximation (4.17) on the outermost closed contour yields, noting that $q$ in (4.17b) is $f_{n} / H$, 


$$
\begin{gathered}
\Psi_{I}=-\frac{\tau_{m}}{\rho \beta R}\left[x_{e}\left(y_{N}\right)+2 \frac{R^{2}}{\left(y_{N}-y_{S}\right)} \sin ^{-1}\left(y_{N} / R\right)+\frac{2}{3} \frac{x_{T}^{2}}{\delta_{s}}\left(\frac{f_{o}}{f_{n}}\right)^{3}\right], \\
\delta_{s} \equiv \frac{\lambda}{\beta H}
\end{gathered}
$$

where $\delta_{S}$ is Stommel's bottom friction boundary layer thickness.

Note that were $x_{T}=0$, i.e. if there were no skirt, (4.23) would yield the island constant as given in PPSH. The island constant in the presence of the skirt shows the enhancement of the circulation by the skirt by the addition of the third term in (4.23a). Of course, this term assumes that lateral friction is negligible on the western edge of the skirt with respect to bottom friction. Assuming that is the case, the more complete formula for the variation of $\psi$ on the skirt, (4.16), together with (4.22) yield 1.02 Sverdrups for $\psi_{T}$ and 1.379 Sverdrups for $\Psi_{I}$ if we choose $\delta_{S}$ of $27 \mathrm{~km}$ (the width of the Stommel layer in our calculation). The numerical calculation for the linear case of Figure 5 yields slightly greater than 1.36 Sverdrups for $\Psi_{I}$. The analytical result is, thus, in surprisingly good agreement with the numerical calculation when we consider that we have ignored the effect of lateral friction.

\section{b. The western meander}

We turn now to the remarkable inverted $\mathrm{V}$ shape of the streamlines emerging from the western limit of the skirt that form the large stationary meander at the western edge of the skirt. Consider the wedged shape region in Figure 12 that is carved out of the western skirt by the lines of ambient potential vorticity that do not close on themselves. The region is defined by the ambient $q$ isoline that starts in the northwest corner of the skirt and that intersects the southern edge of the skirt at $x=-x_{T}+d$ where

$$
\frac{d}{x_{T}}=\frac{\beta\left(y_{n}-y_{s}\right)}{f_{n}}<1
$$

by hypothesis. In the small wedge described above we note that the $q$ contours do not wrap around the island but run from the southern base of the topography to its western edge. On $x=-x_{T}$ and $y=y_{S}$, the latitude of the southern most tip of the extended topography the stream function is given by the Sverdrup stream function at that point, i.e. $\psi_{s}\left(-x_{T}, y_{S}\right)$. We suppose that at the edge of the topography's southern boundary, as it falls to the full depth $H$ as $x=-x_{T}$, the classical Sverdrup solution will hold i.e. that the Sverdrup solution is continuous with the solution on the skirt at $x=-x_{T}$ and $y=y_{S}$ is approached from the flat region. We will heuristically apply the value of the Sverdrup stream function at $\left(-x_{T}, y_{S}\right)$ to that point.

The Sverdrup solution satisfies

$$
\beta \frac{\partial \psi_{s}}{\partial x}=\vec{k} \cdot \nabla \times \vec{\tau} / \rho=\frac{2 \tau_{m}}{\rho R}
$$




$$
x=x_{T}
$$

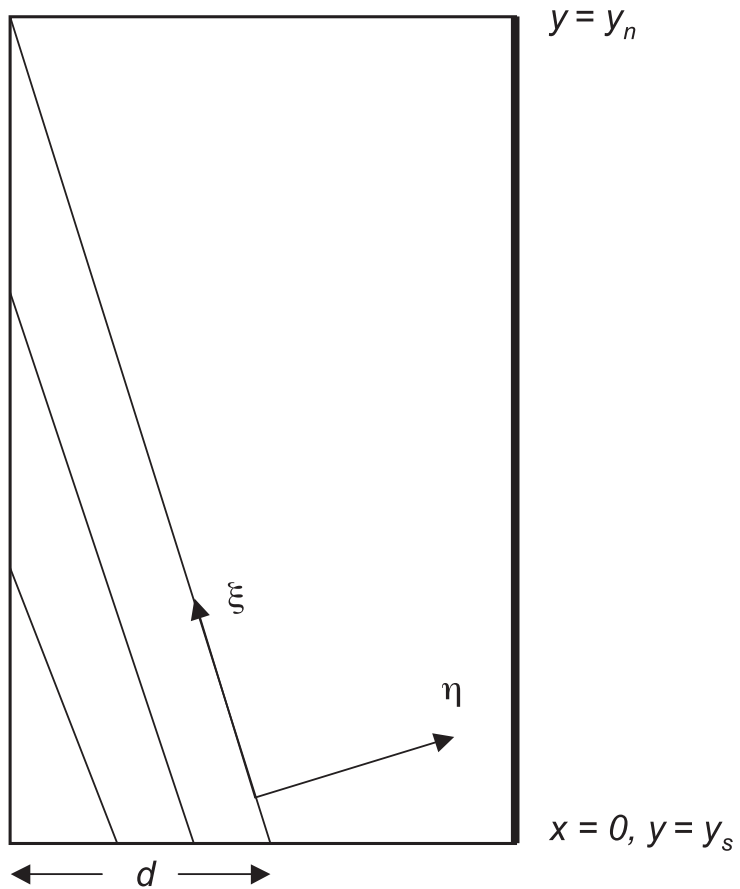

Figure 12. A schematic of the lines of constant ambient $q$ near the western edge of the skirt and the coordinate frame used to describe the western meander.

and applying (2.9a) allows us to write the value of $\psi_{s}$

$$
\psi_{s}\left(-x_{T}, y_{S}\right)=2 \frac{\tau_{m}}{\rho \beta R}\left[-x_{e}\left(y_{S}\right)-x_{T}\right]
$$

while at $x=-x_{T}+d$, on that same latitude the stream function is given by $\psi_{T}$. We suppose further that on $y=y_{s}$, in the small interval, $-x_{T} \leq x \leq-x_{T}+d$ we can approximate $\psi$ as

$$
\psi=\psi_{s}+\left(\psi_{T}-\psi_{s}\right) \frac{x+x_{T}}{d},
$$

where $\psi_{s}$ is the Sverdrup stream function, given by (4.27). We proceed by forming a coordinate system as shown in Figure 12 rotated so that one coordinate $\xi$ is along the $q$ isolines and the other, $\eta$ is perpendicular to it, i.e.

$$
\eta=\left(x+x_{T}\right) \cos \theta+\left(y-y_{s}\right) \sin \theta, \quad \xi=\left(y-y_{s}\right) \cos \theta-\left(x-x_{T}\right) \sin \theta
$$

with its origin at the southwest corner of the topography. From (4.8) it follows that 


$$
\cos \theta=\frac{1}{\left[1+\frac{\beta^{2} x^{2}}{f^{2}}\right]^{1 / 2}} \approx 1, \quad \sin \theta=\frac{\frac{\beta}{q\left(-h_{x}\right)}}{\left[1+\frac{\beta^{2} x^{2}}{f^{2}}\right]^{1 / 2}} \approx \frac{\beta}{q\left(-h_{x}\right)}=\frac{\beta}{f}(-x) \ll 1
$$

and we recall that for $x<0, h_{x}<0$. The linearized vorticity equation, including topography but ignoring dissipation in this region (the eastern side of the western sub-basin) is:

$$
\frac{\beta}{h} \psi_{x}+\frac{f h_{x}}{h^{2}} \psi_{y}=\frac{c u r l \vec{\tau}}{\rho h}
$$

which, rewritten in the potential vorticity coordinate frame, becomes

$$
q h_{x} \psi_{\xi}=\frac{\operatorname{curl} \vec{\tau}}{\rho} .
$$

Note that along the coordinate $\xi, q$ is a constant as of course is both $h_{x}$ and the wind stress curl. We have used the approximations in (4.30) to obtain (4.32).

The homogeneous solution to (4.32) is simply,

$$
\psi=\psi_{h}(\eta)
$$

and this solution preserves potential vorticity along the $q$ isolines. We use the homogeneous solution in (4.33) to satisfy the boundary condition on $y=y_{s}$ namely, (4.28) or

$$
\psi_{h}=\psi_{s}+\left(\psi_{T}-\psi_{s}\right) \frac{\eta}{d \cos \theta}
$$

which in terms of our original coordinates is

$$
\psi_{h}=\psi_{s}+\frac{\psi_{T}-\psi_{s}}{d}\left[x+x_{T}+\left(y-y_{s}\right) \tan \theta\right] .
$$

We must add a particular solution of (4.32) to (4.35) to take account of the wind curl forcing. The solution which satisfies (4.32) (note that $q$ is a constant along lines of constant $\xi$ ) and is zero at $y=y_{s}$, is

$$
\psi_{p}=\frac{\operatorname{curl} \vec{\tau}}{\rho h_{x} q}[\xi+\eta \tan \theta]
$$

where the second term in the square bracket is an additive constant (as far as (4.32) is concerned) and satisfies the condition that $\psi_{p}$ is zero at $y=y_{s}$. The complete solution is the sum (4.35) and (4.36) in terms of which the zonal velocity at $x=-x_{T}$ can be easily calculated. The particular solution (4.36) contributes a term of order $\frac{\beta x_{T}}{f} \ll 1$ in comparison with the homogeneous solution, reflecting the fact that it measures the cross potential 
vorticity gradient flow driven by the wind stress curl and this is relatively small over steep topography. We then obtain, using (2.1), (4.25), (4.29), and (4.35) after some algebra and using the symmetry of the island so that $y_{N}=-y_{S}$,

$$
u=\frac{\tau_{m}}{\rho \beta H R}\left[\frac{2 R^{2}}{\left(y_{N}-y_{S}\right)} \sin ^{-1}\left(y_{N} / R\right)-\left\{x_{e}\left(y_{N}\right)+2 x_{T}\right\}\right] \frac{f_{n} f_{s}}{f^{2}\left(y_{n}-y_{s}\right)} .
$$

On $x=-x_{T}$ the Sverdrup balance holds and, with the bottom flat there, the meridional velocity just west of $-x_{T}$ is

$$
v=\frac{\operatorname{curl} \vec{\tau}}{\rho \beta H}=\frac{2 \tau_{m}}{\rho \beta H R}
$$

so that the slope of the streamline just to the west of the western boundary of the skirt is

$$
\frac{v}{u}=\frac{\left(f^{2} / f_{n} f_{s}\right)\left(y_{n}-y_{s}\right)}{\left[\frac{R^{2}}{\left(y_{N}-y_{S}\right)} \sin ^{-1}\left(y_{N} / R\right)-0.5 x_{e}\left(y_{N}\right)-x_{T}\right]} .
$$

It is important to note that this result, which determines the sharpness of the inverted $\mathrm{V}$ streamlines coming off the western edge of the skirt is independent of the stress (and its sign) and depends only on geometrical factors and the ratio $f_{s} / f_{n}$ although it does vary mildly in $y$ due to the variation of $f$. Using the geometrical parameters of our calculations, (4.39) predicts a slope west of the skirt of 6.91 at $y=0$, the mid latitude of the island in fairly good agreement with the numerical calculation of a bit over 7 . We emphasize the heuristic nature of some of our approximations but it seems clear that the fundamental explanation of much of the structure of the circulation is rather well understood on the basis of this simple theory.

\section{Unsteady solutions: numerical results}

A thorough analysis of the numerical and laboratory experiments with strong forcing is still under way, and will be the subject of a future publication. We give here a survey of the main results, to highlight differences with the linear regime just discussed. In particular, since in the "standard" experiments (anticyclonic forcing, 200-km wide skirt) we have found unsteady solutions for strong enough forcing, here we seek to determine the threshold for this transition, as a function of the skirt width, and to verify whether a similar threshold exists in the case of cyclonic forcing.

Before addressing these issues, it is interesting to briefly examine the effect of strong forcing on the transports around the island. In Figure 13, we compare the mean total transport to the east of the island $T_{I}$, and the mean transport to the east of the bounding $\mathrm{f} / \mathrm{H}$ contour $T_{T}$, with the corresponding analytic measures $\psi_{I}$, and $\psi_{T}$, derived in the previous section from linear theory (the transports are for standard numerical experiments with different values of $\delta_{I} / \delta_{M}$ ). The figure shows that the ratios between the numerical 

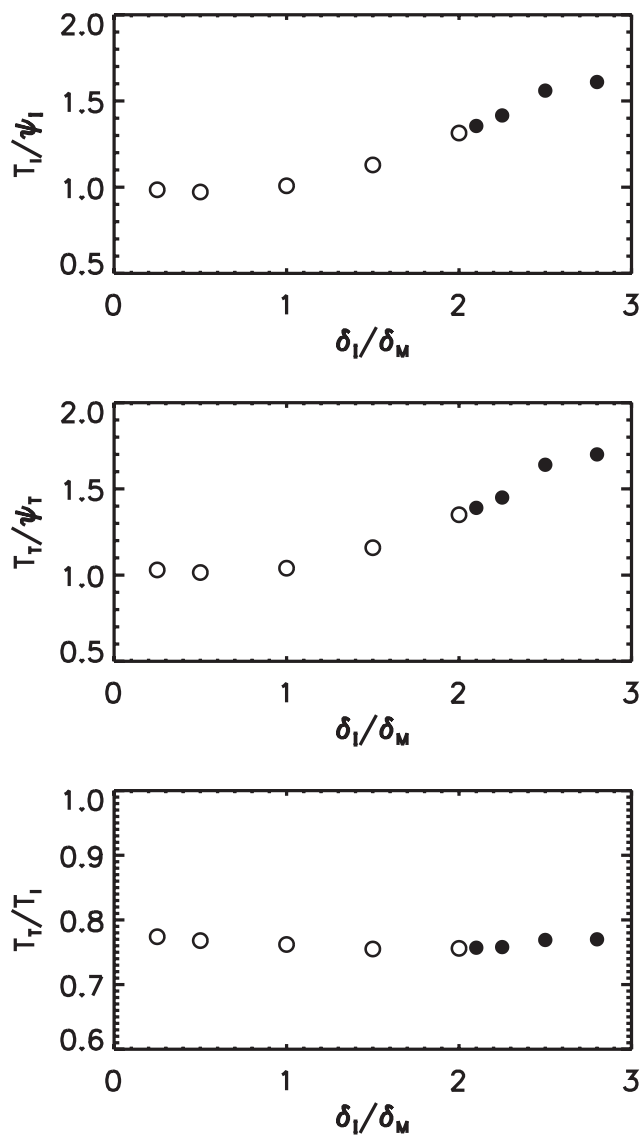

Figure 13. (a) Mean transport to the east of the island $T_{I}$ scaled by $\psi_{I}$ vs. $\delta_{I} / \delta_{M}$. (b) Mean transport east of the bounding closed $f / h$ contour $T_{T}$ scaled by $\psi_{T}$. (c) The ratio $T_{T} / T_{I}$. The filled circles indicate unsteady solutions.

transports and the analytic estimates remain close to unity for $\delta_{I} / \delta_{M}<1$, but then start to increase when moving further into the nonlinear regime. Since the Island Rule integral, (2.10), can be interpreted as a budget of vorticity in the region to the east of the island, with the left-hand side describing the divergence of the planetary vorticity flux between the bounding latitudes of the island, and the right-hand side describing sources and sinks of vorticity, the deviation from the linear estimates indicates that the nonlinear (steady or unsteady) flux of relative vorticity becomes increasingly important when the forcing is increased. On the other hand, the ratio $T_{T} / T_{I}$ remains approximately constant, even in the unsteady solutions, indicating that the closed circulation over the skirt is not strongly affected by the time-dependent dynamics. However, it is important to note that in a more eddy-rich regime, as might occur for a baroclinic flow, this could alter due to potential 


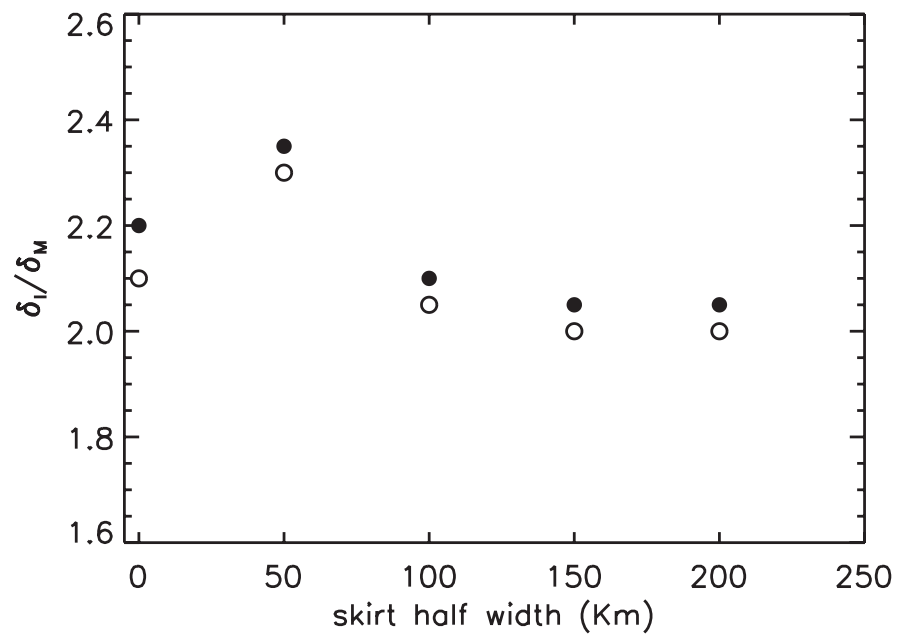

Figure 14. Threshold in $\delta_{I} / \delta_{M}$ for time dependence as a function of the skirt width. The filled (empty) circles unsteady (steady) solutions.

enhanced potential vorticity fluxes across the contour encircling the outermost closed $q$ contour.

Let us now come to the transition issue. Results for the anticyclonic case are summarized in Figure 14, where open circles denote steady solutions, while the filled ones correspond to time-dependent solutions, exhibiting sizable, regular oscillations of the prognostic fields. The transition to unsteady behavior occurs a little above $\delta_{I} / \delta_{M}=2$, the exact threshold lying in the range 2.1-2.2 in the no-skirt case, between 2.3 and 2.35 for a $50 \mathrm{~km}$ wide skirt, and just above 2.0 for skirts of width of $100 \mathrm{~km}$ or larger. In experiments with no island (not shown here) the transition is found to occur at a slightly lower value of the forcing around $\delta_{I} / \delta_{M}=1.9$. Overall, these results indicate that, in the case of anticyclonic forcing, the threshold for unsteady behavior is not strongly affected by the presence of an island, with or without a topographic skirt. A possible exception is the case of very narrow skirts, that appear to have a stabilizing effect. This regime of transition between no-skirt and finite skirt (by that meaning a skirt of width definitely larger than the dissipative layer width) will be examined in more detail elsewhere.

The case of cyclonic forcing looks dramatically different. While the simulations with no-skirt, and with no-island, exactly yield the same thresholds found in the anticyclonic case, those with a standard skirt of $200 \mathrm{~km}$ remain perfectly steady well above $\delta_{I} / \delta_{M}=2$. We find that in this case the transition to unsteady behavior occurs at a much higher value of $\delta_{I} / \delta_{M}$, lying between 4 and 5 . As pointed out in Section 3, this striking asymmetry is due to the presence of topographic Rossby waves on the skirt, that are held stationary by the cyclonically forced circulation, and strongly alter the structure of the surrounding flow.

We now return to the case of anticyclonic forcing to further explore the time-dependent dynamics. The stream functions of the experiments above threshold exhibit regular 


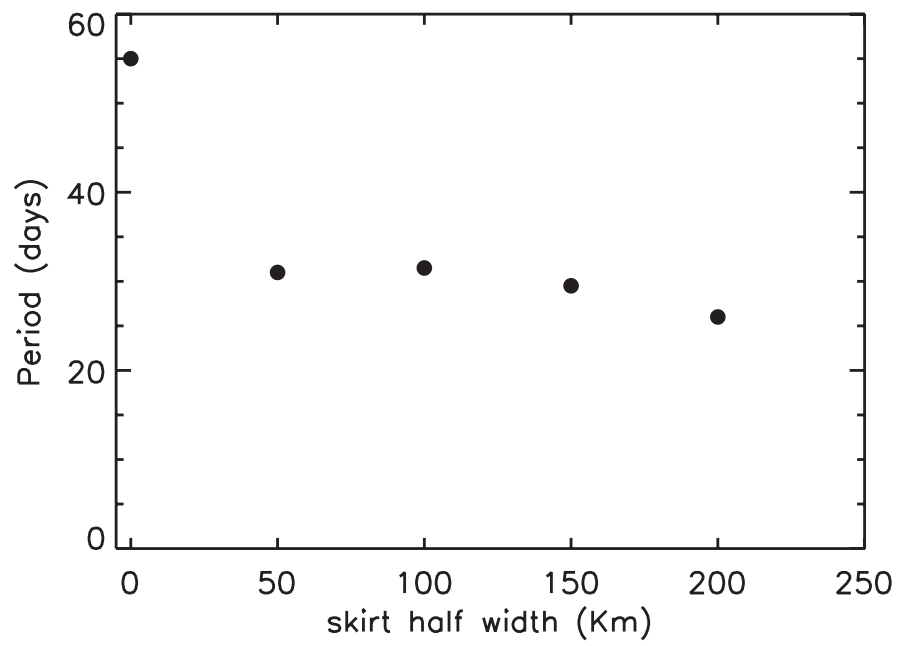

Figure 15. Oscillation periods (in days) for the unsteady solutions of Figure 14.

oscillations, of wider amplitude in the eastern part of the basin (the corresponding periods are given in Fig. 15). These oscillations are associated with periodic formation and shedding of cyclonic vortices in the northeastern quadrant of the basin. An example is given in Figure 16 for an experiment with standard skirt and $\delta_{I} / \delta_{M}=2.1$. The figure shows snapshots of the stream function during an oscillation period, indicating the presence of an instability of the narrow meander that the western boundary current forms after separating from the basin boundary (see the first frame, day 902). At day 908, a small cyclonic vortex has been shed from the inner part of the meander, while a companion anticyclonic vortex has appeared more to the east. After a few days, the two vortices have evolved into a large dipole (see the third frame), with the cyclonic pole slowly moving southward. The dipole lasts for several days, until the cyclone crosses the mid-latitude line and starts to be dissipated (fourth frame), ultimately leading to the restoring of the initial pattern.

We find this to be a common behavior; we observe similar dynamics in runs with different skirt widths, and even in absence of a skirt. It should be noted that the development of the instability does not seem to involve the flow in the northern part of the western boundary current, which is almost unperturbed. This is illustrated in Figure 17, where we plot contours of the maximum local variation of the stream function over an oscillation period. The figure shows that the maximum variability is concentrated along the path of the cyclonic vortex, with only a small signal propagating into the western part of the basin through the southern opening, and very weak changes occurring in the western boundary current. Later on, we will see that similar features emerge in laboratory experiments showing time-dependent dynamics (see, in particular, Fig. 23).

There are, however, important aspects of the time-dependent dynamics that are strongly 

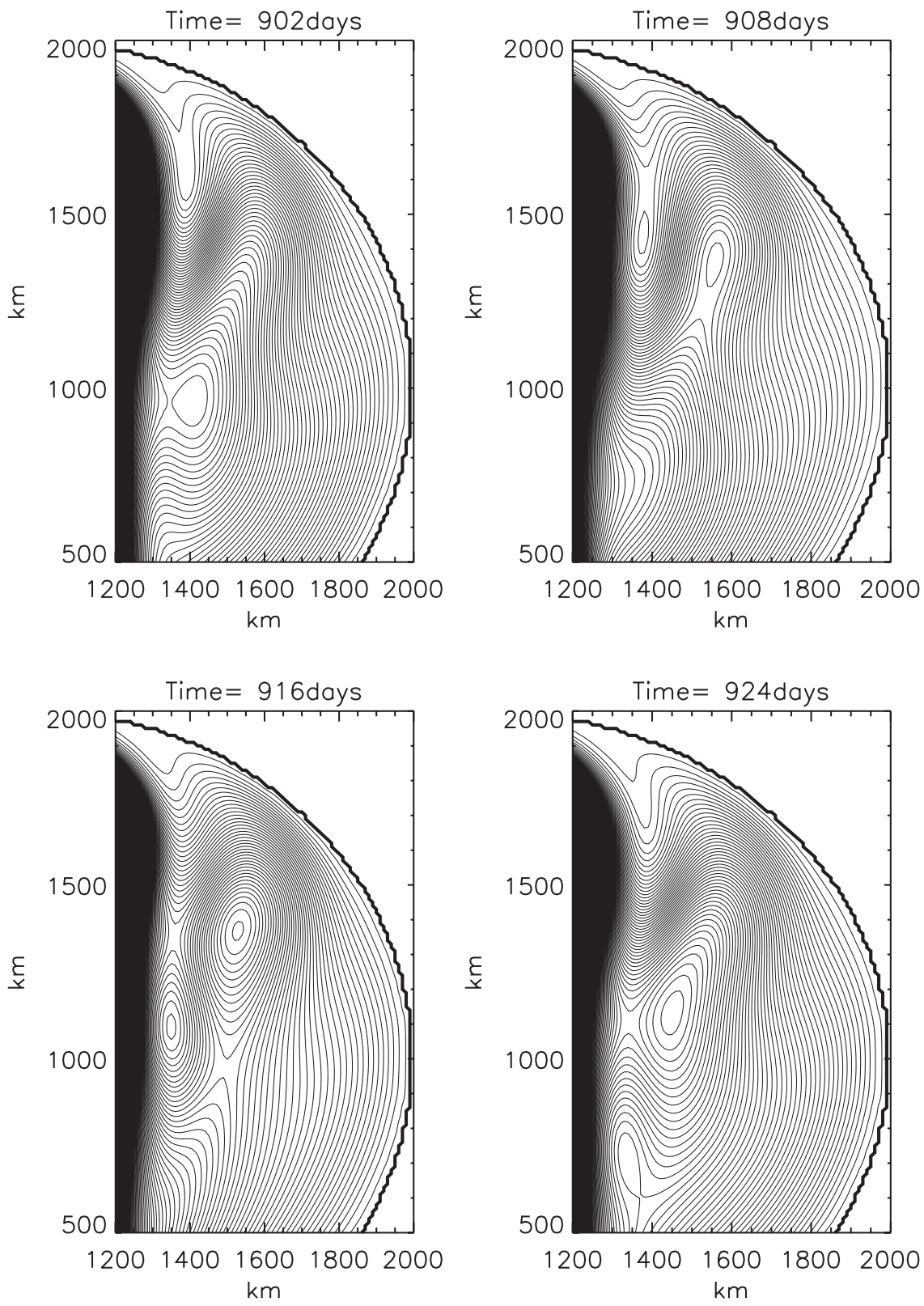

Figure 16. Snapshots from an unsteady run with $\delta_{I} / \delta_{M}=2.1$ and a 200-km skirt, illustrating the development of an instability of the large meander in the northeastern quadrant. The instability period is of about 26 days. 


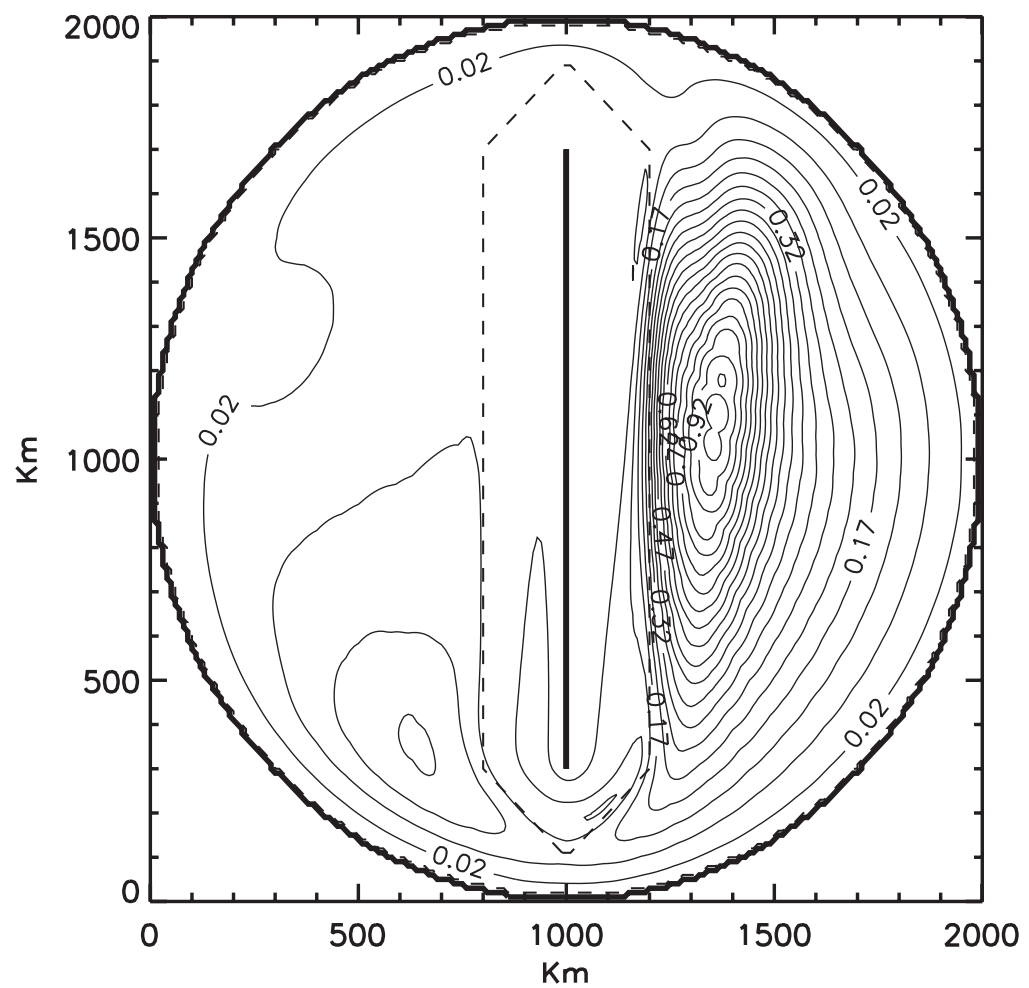

Figure 17. Maximum local variation of the stream function for the run of Figure 16, scaled by its maximum value. The largest variations occur along the path of the cyclonic vortex periodically shed from the northeastern meander.

affected by the presence of a skirt. As shown in Figure 15, the oscillation periods just above threshold do not vary much with the skirt width (they slightly decrease, from about 31 days to 26 days, for skirt widths going from $50 \mathrm{~km}$ to $200 \mathrm{~km}$ ), but they are all much shorter than the 55-day period found in the no-skirt case. To get some insight about the causes of this difference, in Figure 18 we compare the stream functions, averaged over an oscillation period, for two runs with similar forcing, the no-skirt run with $\delta_{I} / \delta_{M}=2.2$ (top panel) and the standard skirt run with $\delta_{I} / \delta_{M}=2.1$ (lower panel). We focus on the northeastern quadrant of the basin, where the instability develops. It is seen from the figure that the meanders formed by the western boundary currents are similar in shape and strength in the two cases, the main difference being the orientation, which is more meridional when a skirt is present (in the no-skirt case, the meander is tilted in the northeastern direction, due to the presence of a recirculation on the eastern side of the island that deflects the westerly flow, and to the fact that separation from the boundary occurs earlier, in the northwestern quadrant). Since we are just above threshold in both cases, the similar shapes and strengths of the meanders suggest a mechanism of shear instability related to the structure of the flow 


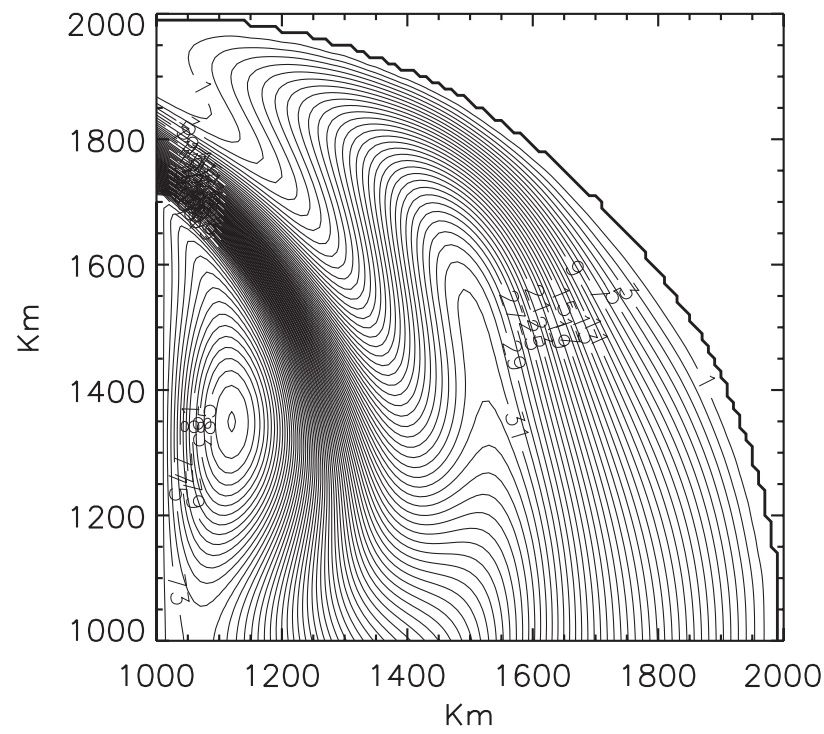

a)

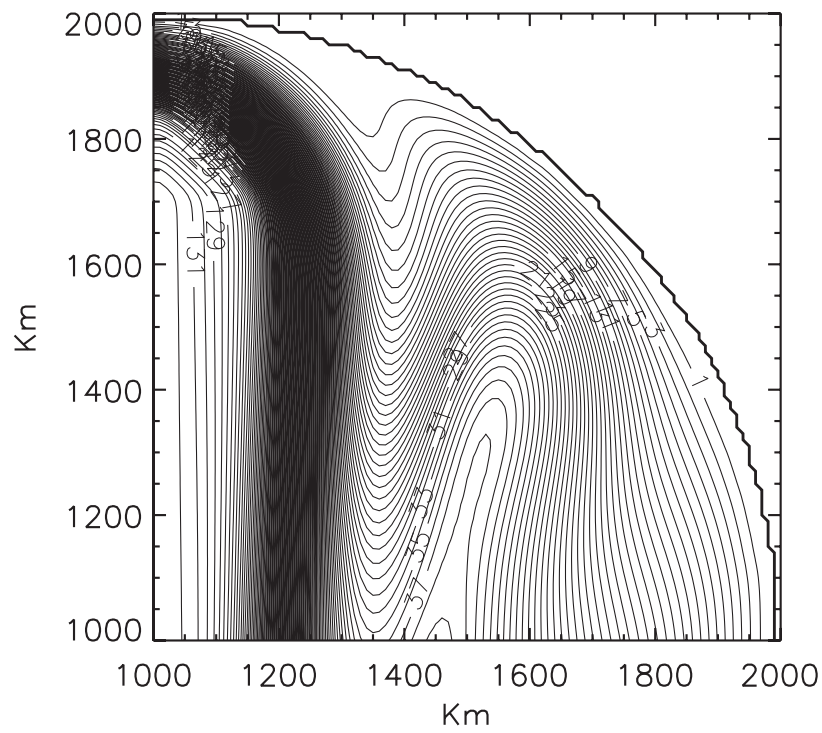

b)

Figure 18. Zooms of the average stream function of (a) a run with $\delta_{I} / \delta_{M}=2.2$ and no skirt and (b) the run Figure 16. The two runs are just above the instability threshold. Shapes and strengths of the meanders are similar, but the instability period is much shorter for (b).

in the meanders. This looks different from the picture drawn in several works exploring the nonlinear dynamics of flows in enclosed basins not containing islands (see Griffiths and Kiss (1999) and references therein), in which the transition to oscillating behavior is attributed to shear instability of the western boundary current itself. 
That both types of instability are possible has been shown by Meacham and Berloff (1997), who have investigated numerically the stability of wind-driven, single layer steady flows in rectangular basins, finding several classes of normal modes, characterized by different locations of the region of dominant transfer of energy between the basic flow and the perturbation. The instability we have previously described appears similar to one of the "interior" modes considered by Meacham and Berloff (1997), observed in a regime of strong forcing, in which the region of dominant energy exchange is located in the vicinity of a meander formed by the southern boundary current (cyclonic forcing is used in that work).

Figure 18 also suggests that the difference of oscillation periods in the two simulations is bound to be related to the presence of an intense dissipative boundary layer across the whole eastern boundary of the skirt, directly fed by the western boundary current. This boundary layer gives rise to a strong southward flow in the region just to the west of the meander, that can entrain toward the south the cyclonic anomaly much more efficiently than in the no-skirt case (in the latter case there is a much weaker southward flow, more confined meridionally). This makes possible a shorter cycle of the instability in the skirted case.

The different spatial structures of the modes are highlighted in Figure 19 which shows the stream function anomalies, at times corresponding to the initial phases of the instability cycles; i.e., when the cyclonic vortices have just been shed. It is seen that the strengths of the perturbations are similar, while the spatial patterns differ considerably; the anomaly is practically confined in the northeastern quadrant in the no-skirt case (and basically remains so at following times), while it involves the whole eastern basin in the case with standard skirt, with a weak signal propagating in the southwestern quadrant.

Finally, we show in Figure 20 a snapshot from a simulation with standard skirt and $\delta_{I} / \delta_{M}=2.5$. The oscillation period for this run has further decreased to 21 days (it was about 26 days at $\delta_{I} / \delta_{M}=2.1$; the decrease of period when the forcing is increased was found to be a generic feature, both in the simulations with skirts, and in those without). The time evolution is still mostly confined to the eastern side of the basin, and is qualitatively similar to that of Figure 16, except for the fact that now the cyclone formation involves a larger part of the meander, and that a new cyclone is formed well before the previous one was dissipated.

To conclude this brief analysis of the simulations with strong forcing, we point out that some issues should be further investigated before claiming applicability of the previous results to more general contexts. For example, since the main instability mechanism appears related to the structure of the meander, changes in the geometry of the problem (e.g., island meridional extent, distance from the basin boundaries, shape of the boundaries) could significantly affect the instability threshold and the oscillation frequencies, and even introduce additional instability mechanisms. The role of nonlinear fluxes of relative vorticity also deserves further examination. In principle, eddies in the time-dependent cases could flux vorticity across the bounding streamline, $\psi_{T}$, and so change its value as 

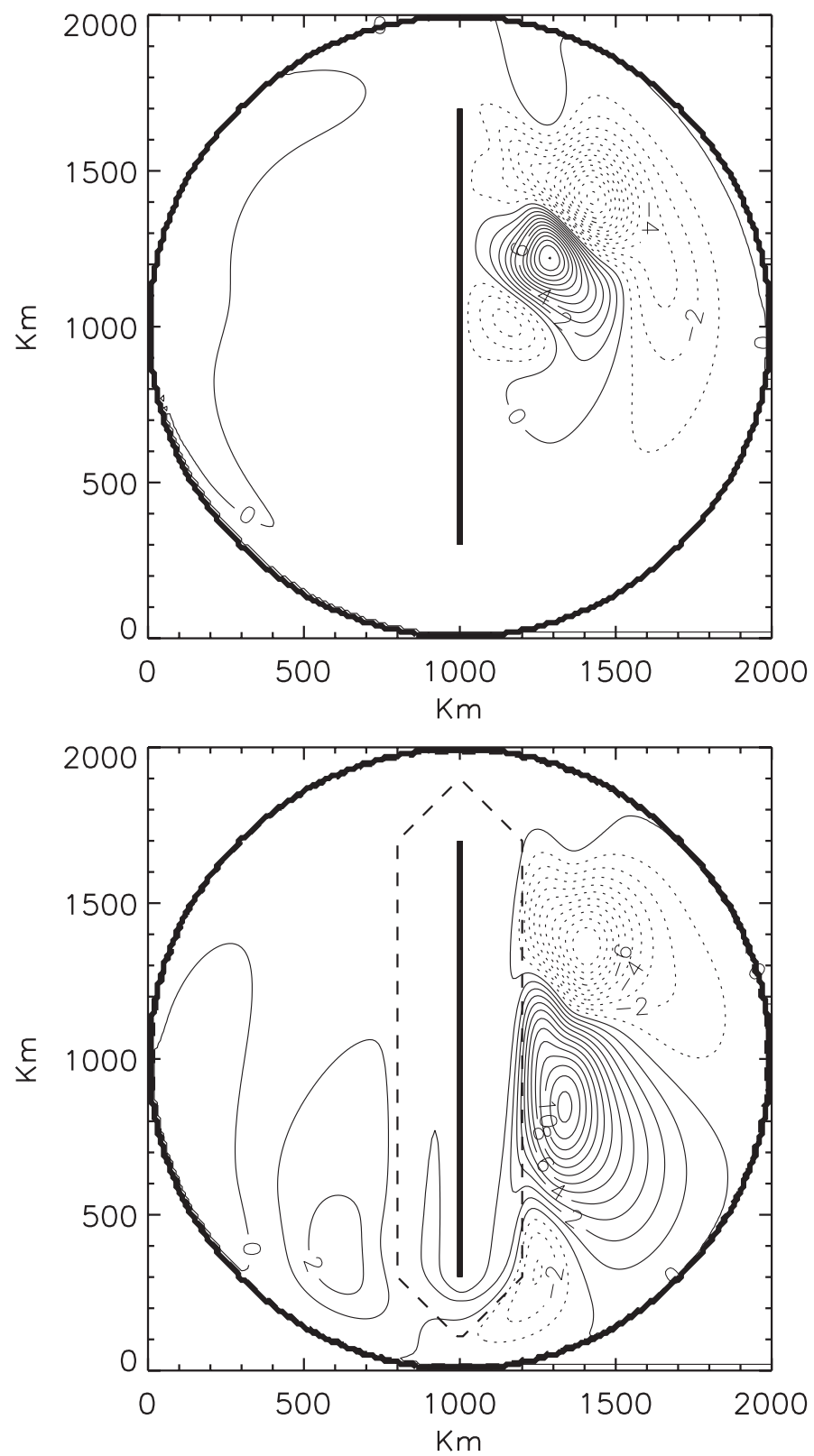

Figure 19. Stream function anomalies for the runs of Figure 18, at times at which the cyclones have just been shed. In the skirted case (b) the perturbation involves the whole eastern basin. 


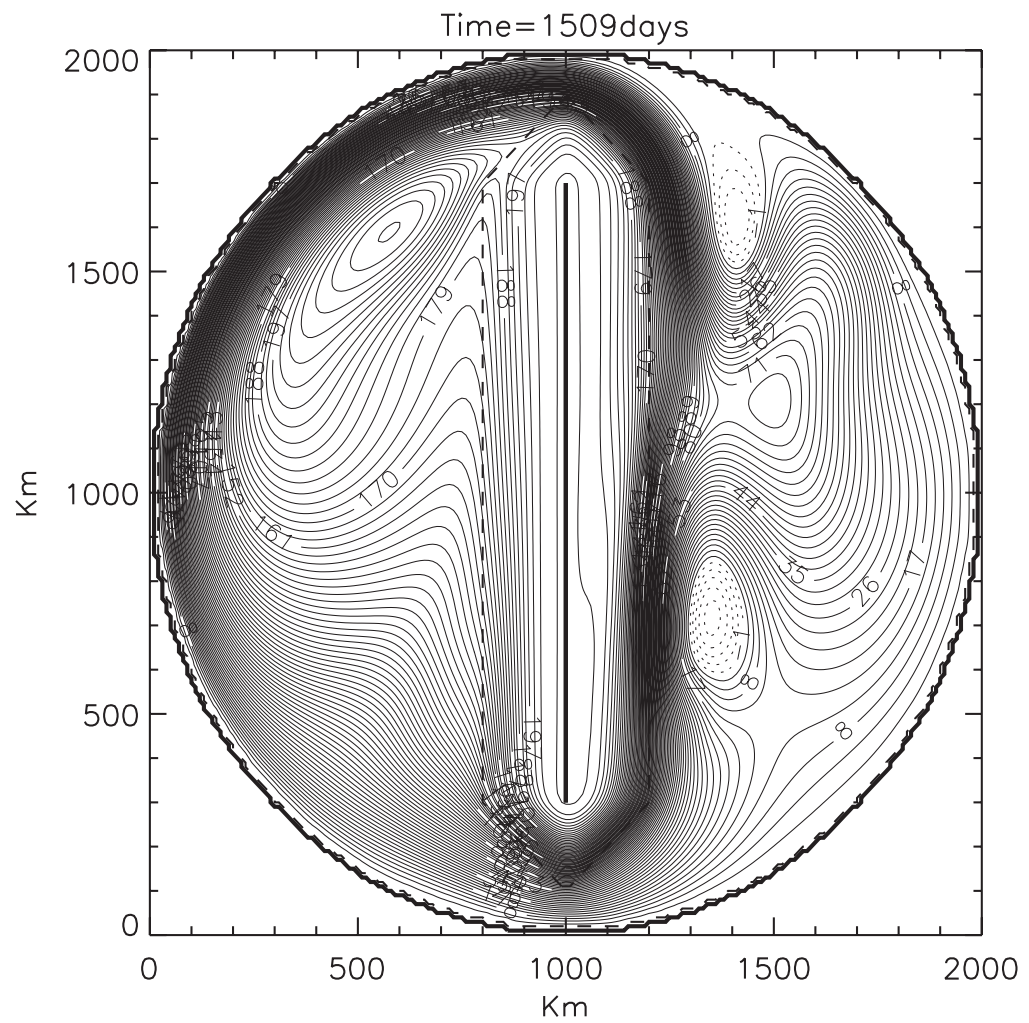

Figure 20. Snapshot from an unsteady run with $\delta_{I} / \delta_{M}=2.5$ and a $200 \mathrm{~km}$-skirt, showing two southward propagating cyclonic vortices in the eastern part of the basin. The oscillation period is of about 21 days.

well as that of $\Psi_{I}$. In the calculations reported here, the eddy field generated to the east of the island either damps away before reaching the western side of the island or so closely follows the topography that the effect we might anticipate does not appear to occur. It does suggest examining future cases with much reduced friction to see if an enhanced eddy flux can lead to more significant alterations in these constants and hence to the fluid interchange between the sub-basins or to the fluid trapped to the topography.

\section{Laboratory experiments}

In previous studies of large-scale flow with (PPSH; Helfrich et al., 1999) and without topography (e.g. Beardsley, 1969; Griffiths and Kiss, 1999) laboratory experiments have proven very informative. They allow study of the nonlinear regime not easily accessible with theory and, while limited by viscous and scale effects, they serve as stringent tests of numerical models, particularly when new or novel flows occur. Thus we have preformed a series of experiments to simulate the flows discussed in the previous sections. 


\section{a. Setup and parameters}

The laboratory experiments are based on the lid-driven, sliced-cylinder analogue of wind-driven circulation on the $\beta$-plane (Pedlosky and Greenspan, 1967; Beardsley, 1969). The apparatus used in these experiments is the one used in Helfrich et al. (1999). It consists of a cylindrical Plexiglas tank of radius $R=21.4 \mathrm{~cm}$ fitted with a uniformly sloping bottom with slope $s=0.15$. Equivalent north ( $y$ positive) is toward the shallow end of the tank. The tank is placed on a precision direct-drive turntable which was rotated in a counter-clockwise direction (viewed from above) with angular frequency $\Omega=f_{o} / 2=1.5 \mathrm{~s}^{-1}$. The tank is filled with fresh water with mean depth $H=$ $20.4 \mathrm{~cm}$. The flow is forced by a horizontal plate glass lid that is rotated, via a stepper motor-driven belt, at frequency $\Delta \Omega$ relative to the table. Both anticyclonic, $\Delta \Omega<0$, and cyclonic, $\Delta \Omega>0$, forcing is considered.

The island is fabricated from plastic. It has a uniform slope $s_{I}=4.7$, is $30.5-\mathrm{cm}$ long and $0.5-\mathrm{cm}$ wide at the top and is and oriented in the north-south direction in the center of the tank. There is a $0.1-\mathrm{cm}$ gap between the top of the island and the lid. The north and south ends are semi-circular in plan view in contrast to the triangular shape of the island in the numerical solution. The topographic contours are shown in Figure 21a. The ratio of the width of the skirt to the tank radius is 0.2 .

Digital particle image velocimetry (DPIV) is used to obtain quantitative information on the flow. A 2-mm thick horizontal light sheet from a pulsed Nd:Yag laser is positioned $6.7 \mathrm{~cm}$ below the upper glass lid. The light sheet enters the tank at the northern end. Other than a thin pie-shaped shadow region on either side of the island, the gap just to the south of the island, and the outer $\approx 0.5 \mathrm{~cm}$ of the tank that was obscured by the lid mechanism, the whole domain is illuminated. Small plastic particles (Vestostint 5155) with mean diameter of $10 \mu \mathrm{m}$ and density $1.016 \mathrm{gm} \mathrm{cm}^{-3}$ are used for the DPIV tracer particles. The flow is imaged from above with a LaVision Imager proX 4M $(2048 \times 2048$ pixels $)$ digital video camera. Typical times between PIV image frames are relatively long, 0.2-0.333 s, since the flow is quite slow $\left(\approx 0.1 \mathrm{~cm} \mathrm{~s}^{-1}\right)$ outside of the western boundary layers. The image sequences are processed with the LaVision's DaVis software using a multipass DPIV method that ends with $32 \times 32$ pixel interrogation boxes with $50 \%$ overlap in both directions. Each final PIV interrogation regions corresponds to a box of approximately $6.5 \mathrm{~mm}$ on a side. Post-processing for removal of bad vectors is done. The field of view is fully calibrated to correct for optical distortion.

The frictional Munk and Stommel boundary layer scales are given by $(2.5 \mathrm{a}, \mathrm{c})$ with the lateral friction coefficient $A=v=0.01 \mathrm{~cm}^{2} \mathrm{~s}^{-1}$, the kinematic viscosity of fresh water, and the bottom friction coefficient

$$
\lambda=H f_{0} E_{v}^{1 / 2}, \quad E_{v}=\frac{v}{\Omega H^{2}} .
$$

Contributions to $\lambda$ from the top and bottom Ekman layers are included. The background topographic beta parameter is 
a) $\delta_{1} / \delta_{M}=0.98$

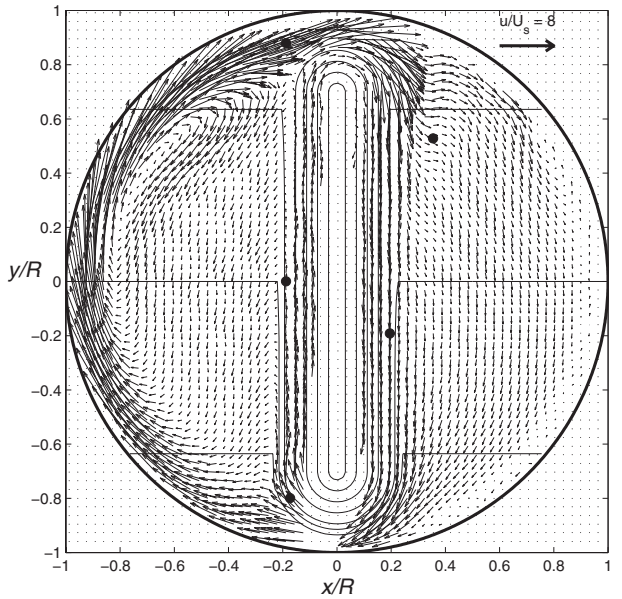

b) $\delta_{1} / \delta_{M}=2.00$

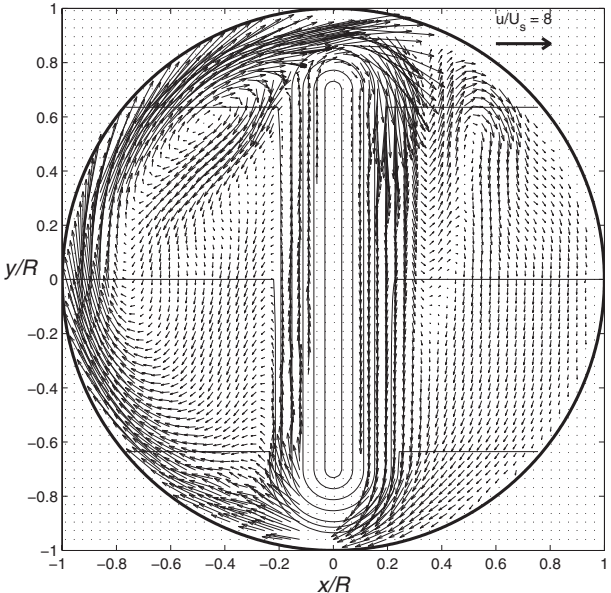

c) $\delta_{\mathrm{I}} / \delta_{\mathrm{M}}=2.81$

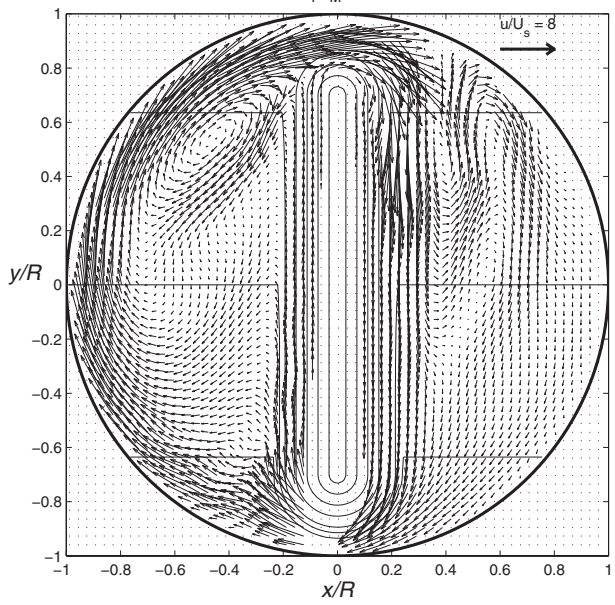

Figure 21. Mean velocity vectors scaled by $U_{S}$ for anticyclonic forcing at (a) $\delta_{I} / \delta_{M}=0.98$, (b) 2.00 , and (c) 2.81. Contours of the basin depth $h$, scaled by $H$, are shown for $h / H=0.1-1.1$ in intervals of 0.2. The solid circles in (a) indicate the locations of the time series in Figure 22.

$$
\beta=\frac{f_{0} s}{H} .
$$

For these experiments the Munk thickness $\delta_{M}=0.77 \mathrm{~cm}$ is slightly greater than the Stommel scale $\delta_{S}=0.54 \mathrm{~cm}$ and both are constant.

The surface Ekman pumping due to the differential lid rotation,

$$
w_{0}=\left(\frac{v}{\Omega}\right)^{1 / 2} \Delta \Omega
$$


drives flow in the tank with interior Sverdrup velocity scale

$$
U_{S}=\frac{f_{0}\left|w_{0}\right|}{\beta H}=\frac{\left|w_{0}\right|}{s} .
$$

The experimental control parameter is the ratio of the inertial boundary-layer scale, $\delta_{I}=$ $\left(U_{S} / \beta\right)^{1 / 2}$, to the Munk scale (2.5a). From the relations above this ratio becomes

$$
\frac{\delta_{I}}{\delta_{M}}=\frac{|\Delta \Omega / \Omega|^{1 / 2}}{2^{1 / 6} E_{v}^{1 / 12} s^{2 / 3}} .
$$

For these experiments $\delta_{I} / \delta_{M}$ is varied over the range $0.74-2.81$ for anticyclonic forcing and 1.05-3.50 for cyclonic forcing by changing $\Delta \Omega$.

\section{b. Results}

i. Anticyclonic forcing. An example of the mean flow for a moderately nonlinear anticyclonic experiment with $\delta_{I} / \delta_{M}=0.98$ is shown in Figure 21a. Only half of the computed velocity vectors are shown for clarity (hence are all independent). There is a strong jet that flows through the gap at the northern end of the island to form a standing meander in the northeast quadrant of the basin. Flow on the island flanks follows the topographic (i.e. geostrophic contours) on the upper reaches of the island slope. Along the western side of the island, flow on the outer flank slowly leaks off the island to merge into the Sverdrup interior. The mean flow from a more nonlinear case, $\delta_{I} / \delta_{M}=2.0$, is shown in Figure 21b. The mean velocity field remains qualitatively unchanged. As expected, there is a northward shift and intensification of the western boundary-layer recirculation zone in the northwest quadrant. There is a similar intensification and deepening of the standing meander in the northeastern quadrant. These mean flows in Figures 21a,b are in very good agreement with the nonlinear numerical solutions at nearly the same values of $\delta_{I} / \delta_{M}$ in Figures 7 and 8 a despite minor differences in the topography.

Figure $21 \mathrm{c}$ shows the mean flow for a strongly nonlinear experiment with $\delta_{I} / \delta_{M}=$ 2.81. The meander to the east of the northern gap is again deeper and has larger velocities relative to $U_{S}$ than for $\delta_{I} / \delta_{M}=2.00$, but is otherwise quite similar. However, as in the numerical experiments, the flow for this strongly nonlinear case has become timedependent.

Time series of horizontal velocities at five points in the domain are shown in Figure 22 for $\delta_{I} / \delta_{M}=1.48$ and 2.81. The locations of the time series points are shown in Figure 21a. They sample the flow in the western boundary layer in the northwest quadrant just before the flow enters the northern gap, a point in the northeast quadrant within the meander, on the eastern flank of the island just outside the bounding geostrophic contour, on the island flank just to the west of the southern gap, and a point on the western slope of the island just outside the bounding closed geostrophic contour.

At $\delta_{I} / \delta_{M}=1.48$ (Fig. 22 left column) the flow is steady at all locations except for small fluctuations of about $0.5 U_{S}\left(\approx 0.015 \mathrm{~cm} \mathrm{~s}^{-1}\right)$. From the location in the meander (second 

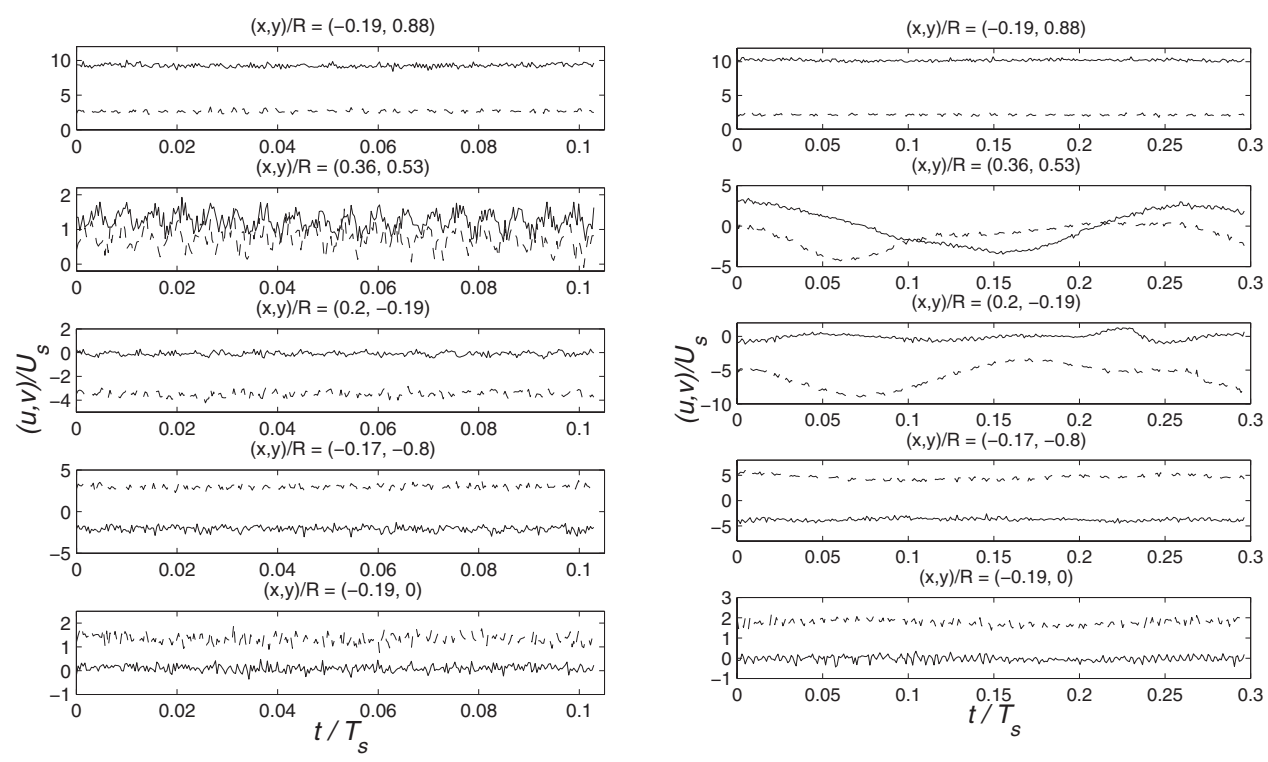

Figure 22. Time series of velocity ( $u$-solid, $v$ - dashed) at five points in the basin for anticyclonic forcing at $\delta_{I} / \delta_{M}=1.48$ (left column) and 2.81 (right column).

panel) there is a dominant period to these fluctuations that is exactly the table rotation period $(4 \pi / 3 \mathrm{~s})$. Apparently, noise from the table drive or errors in table leveling is forcing the fluctuations. These fluctuations are also at the limits of the PIV resolution and correspond to particle displacements of $0.2-0.3$ pixels for the magnification and interframe times employed. Measurements over longer periods than shown in the figure indicate that the flow is steady with the exception of the table oscillation period, which is easily removed by averaging.

The time series records for $\delta_{I} / \delta_{M}=2.81$ are shown in the right column of Figure 22 . The flow in the meander region (second panel) shows significant variation with amplitude of $3 U_{S}$. The period of the oscillation is $\approx 0.26 T_{S}$, where $T_{S}=R / U_{S}$. This period is much greater than the table period and different from the lid rotation period, $0.16 T_{S}$, for the experiments indicating that the variability has an internal origin. On the eastern slope (third panel) the oscillation is apparent, though over the slope the cross-isobath component is greatly reduced indicating only weak penetration of the variability across the bounding geostrophic contour. There is an oscillation in the flow just to the west of the southern gap, though it is very weak in comparison with the variability in the eastern basin. The record in the western boundary layer (top panel) is steady except for the small noise fluctuations. The flow on the western flank of the island (bottom panel) appears to have a very weak oscillation at the period of the meander, but again the amplitude is quite small.

The evolution of the flow over one period of the oscillation is shown in Figure 23 for $\delta_{I} / \delta_{M}=2.81$. The unsteadiness is associated with the formation of a large cyclonic 

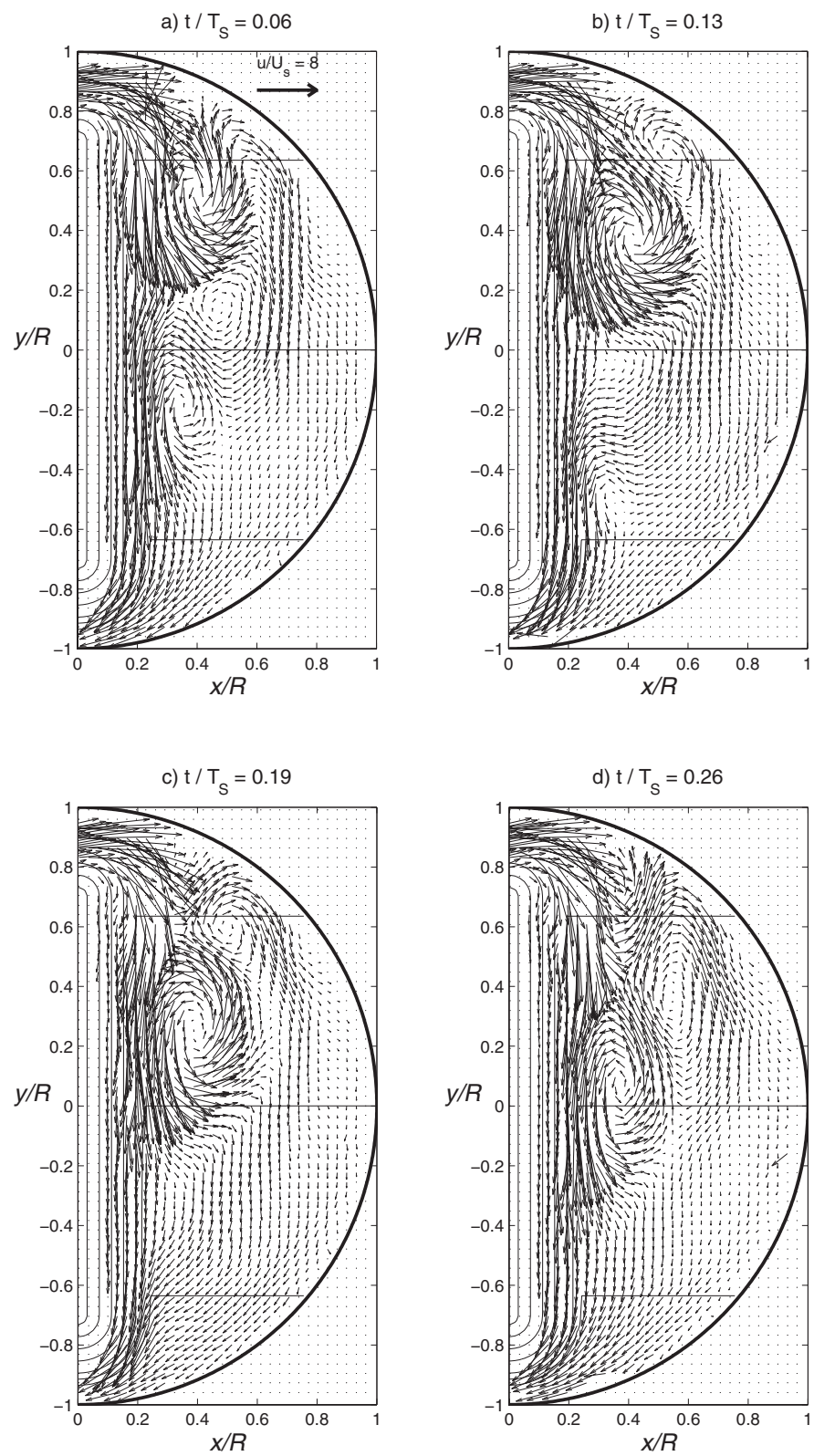

Figure 23. Velocity field in the eastern basin over one period of the oscillation for anticyclonic forcing at $\delta_{I} / \delta_{M}=2.81$.

vortex. The vortex pinches off from the large meander and propagates south along the outer flank of the island. It weakens as it moves southward as shown in Figure 23a where the weaker vortex from the previous cycle is located at $(x, y) / R=(0.35,-0.2)$. By $t / T_{S}=$ 
0.19 this vortex has weakened dramatically and been swept through the southern gap. The Ekman spindown time is $83 \mathrm{~s}$, or $0.4 T_{S}$. This is consistent with the observation that the vortices are strongly damped before they are swept out of the eastern basin with the southward Sverdrup flow. The vortex formation process, southward propagation, and restriction of the variability to the eastern basin outside of the bounding contour is remarkably consistent with the numerical solutions (e.g. Figs. 16 and 17).

The unsteadiness and vortex formation occur for anticyclonic forcing for $\delta_{I} / \delta_{M} \geq 2$. A run at $\delta_{I} / \delta_{M}=1.88$ also exhibited a weak oscillation in the meander region. However, the oscillation period was precisely the lid rotation period. Variability at the lid period also occurred in some cyclonic forcing cases and suggests that the lid was either not perfectly flat or aligned. The period of the oscillation measured in the meander region is shown in Figure 24. An experiment with $\delta_{I} / \delta_{M}=1.81 \mathrm{did}$ not exhibit noticeable variability. The precise location of the transition to time dependence is difficult to determine with confidence given the experimental uncertainty; however, it is clear that the flow is time dependent at a frequency different from the table or lid frequencies for $\delta_{I} / \delta_{M} \geq 2$. Again, this transition to time dependence at $\delta_{I} / \delta_{M} \approx 2$ is consistent with the nonlinear numerical calculations which gave a threshold of 2.0-2.1 for the 200-km island (same ratio of island with to basin radius as the experiments). The rather small difference is likely due to differences in the topography and experimental noise and uncertainty.

A close-up view of the mean velocity field along the western slope of the island is shown in Figure 25a for $\delta_{I} / \delta_{M}=1.75$. The flow inshore of the bounding geostrophic contour (that runs along $x / R=-0.185$ ) is very nearly parallel to the depth contours. Outside this bounding contour the flow clearly leaks off the slope as it moves northward as in the theory and numerical runs. At the northern end of the island $(y / R>0.6)$ some of the fluid flowing higher up on the flank merges with the recirculation gyre of the western boundary layer and flows offshore (also see Fig. 21). Figure 25b shows dye released at mid-depth about one third of the way up the slope at the southern end of the island for an experiment with the same $\delta_{I} / \delta_{M}=1.75$. The offshore leakage of fluid and the sharp turn as the fluid joins the western basin Sverdrup flow is clear. The sharp turn can be clearly seen in a film of the experiment at http://www.whoi.edu/home/video/is03.mov.

ii. Cyclonic forcing. The mean velocity fields with cyclonic forcing for $\delta_{I} / \delta_{M}=1.05$, 2.50, and 3.50 are given in Figure 26. The flow at moderate nonlinearity $\delta_{I} / \delta_{M}=1.05$ is steady and approximately the same (after rotating about $y=0$ ) as the anticyclonic experiment in Figure 21a. The cyclonic case does not exhibit the small meander in the southeast quadrant that exists in the anticyclonic experiment (in the northeast quadrant). When the forcing in increased to $\delta_{I} / \delta_{M}=2.50$ (Fig. 26b) a deep meander emerges in the southeast quadrant and a weak recirculation cell appears near $y / R=0$ just to the east of the island. Both features are in excellent agreement with the numerical solution shown in Figure 9. When $\delta_{I} / \delta_{M}=3.50$ (Fig. 26c) the meander in the southeastern quadrant has 


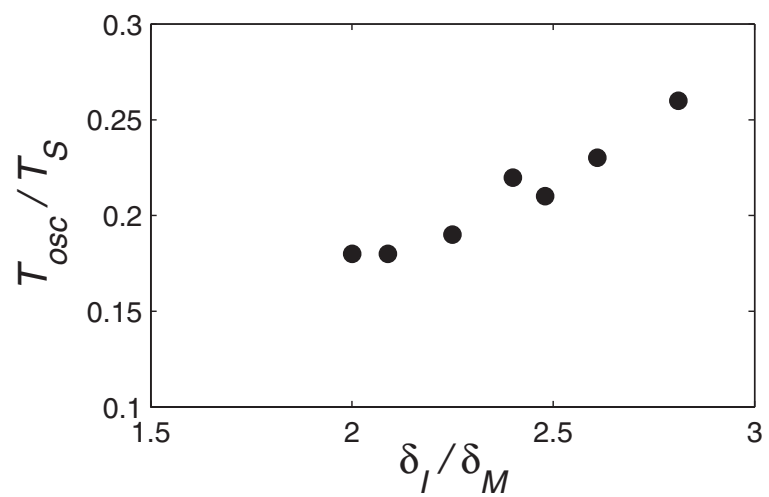

Figure 24. The period of oscillation for the time-dependent anticyclonic forcing runs.

a)

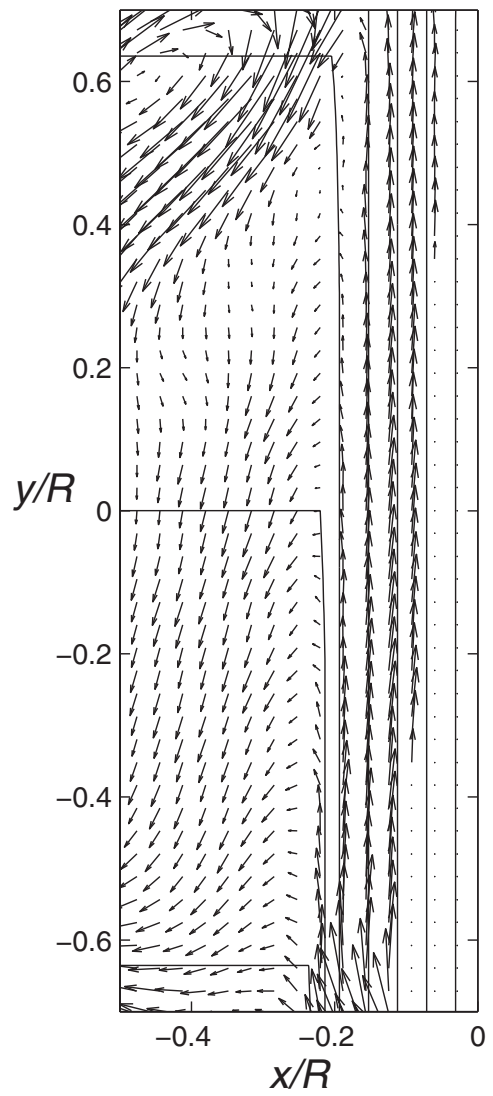

b)

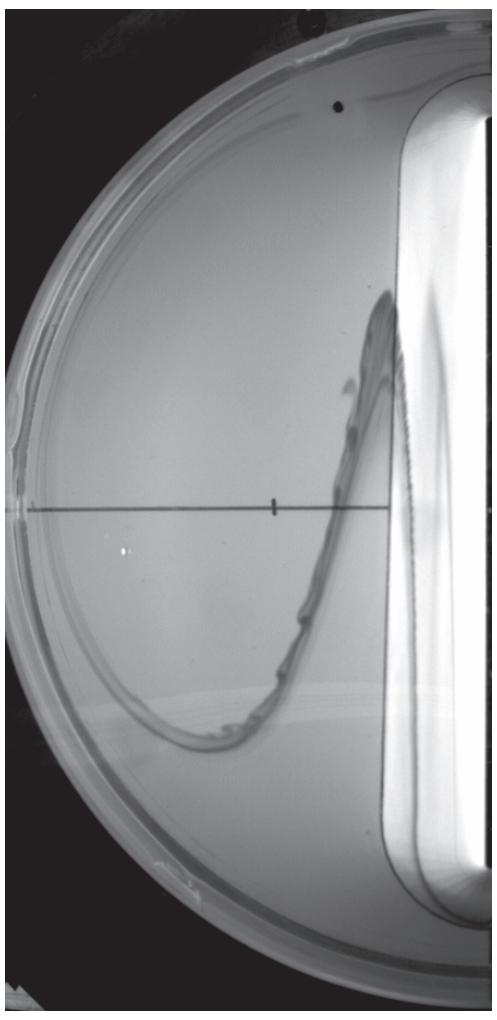

Figure 25. Mean flow on the western slope of the island for $\delta_{I} / \delta_{M}=1.75$. (a) DPIV velocity vectors. The solid lines are the depth contours and the bounding geostrophic contour runs along $x / R=-0.185$. (b) Dye streak released at southern end of island about one third of the way up the slope. 
a) $\delta_{1} / \delta_{M}=1.05$

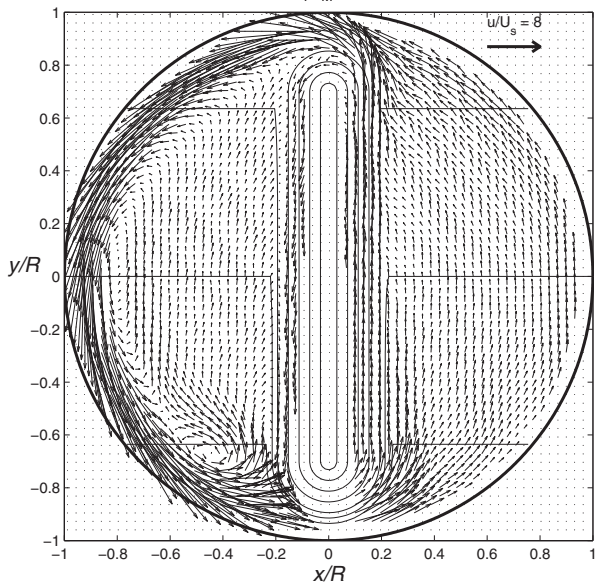

b) $\delta_{1} / \delta_{M}=2.50$

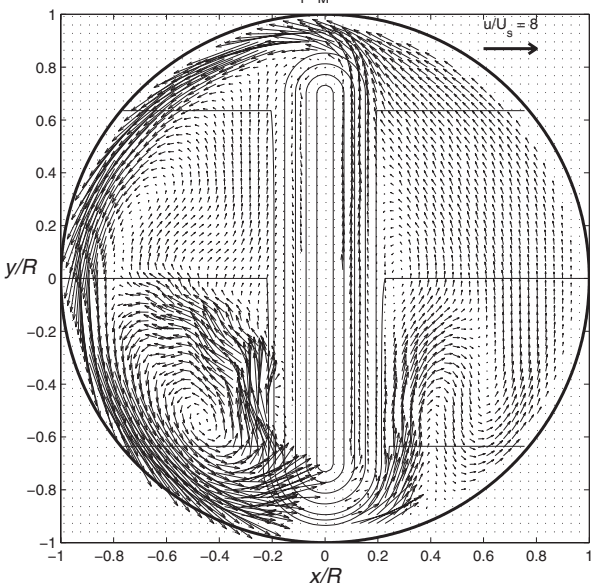

c) $\delta_{1} / \delta_{M}=3.50$

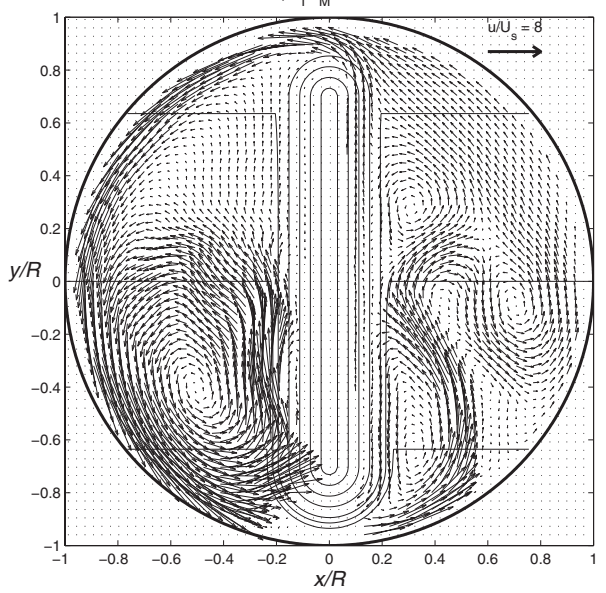

Figure 26. Mean velocity vectors scaled by $U_{S}$ for cyclonic forcing at (a) $\delta_{I} / \delta_{M}=1.05$, (b) 2.50 , and (c) 3.50 .

deepened substantially, the closed recirculation which was near $y / R=0$ has now shifted northward and a new cyclonic recirculation cell has developed just to the east of the island in southeast. As in the numerical runs, these cyclonic experimental runs are steady. There is no evidence of the large-scale variability in the meander and vortex shedding found for the anticyclonic runs with $\delta_{I} / \delta_{M} \geq 2$. Cyclonic forcing for $\delta_{I} / \delta_{M}>3.5$ induced substantial variability in the flow at precisely the lid frequency, suggesting an experimental issue. This prevented useful cyclonic experiments above this value to check the numerical result of a transition to time-dependent flow at $\delta_{I} / \delta_{M}=4-5$. 
(a)

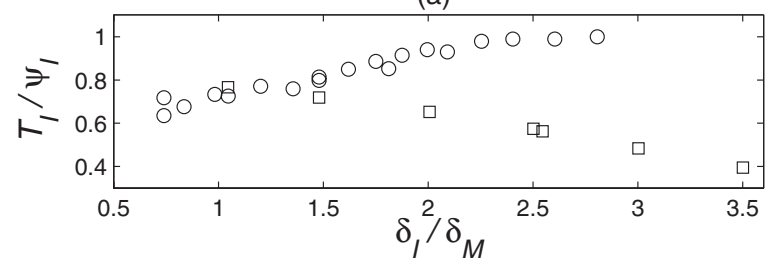

(b)

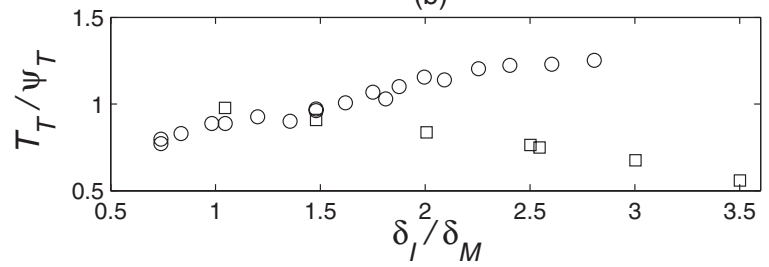

(c)

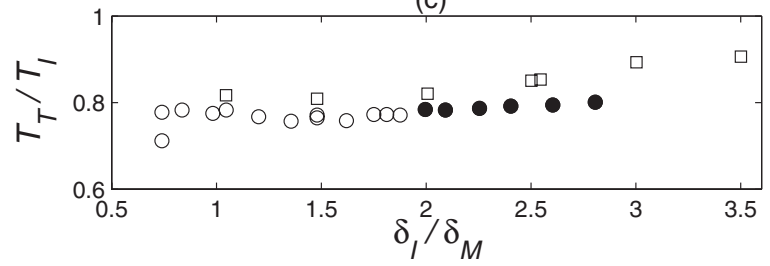

Figure 27. (a) Mean transport to the east of the island $T_{I}$ scaled by $\psi_{I}$ versus $\delta_{I} / \delta_{M}$. The circles (squares) indicate anticyclonic (cyclonic) forcing. (b) Mean transport east of the bounding geostrophic contour $T_{T}$ scaled by $\psi_{T}$. (c) The ratio $T_{I} / T_{T}$. The solid symbols indicate runs with unsteady flow east of the island.

iii. Transport. The total transport to the east of the island $T_{I}$ is evaluated from the mean meridional velocity $v(y)$ and depth $h(y)$ along $x / R=0$. This transport is shown in Figure 27a where it has been scaled by the theoretical linear island stream function, $\psi_{I}$, given by (4.23a). Also shown in Figure 23b is the transport, $T_{T}$, to the east of the bounding closed geostrophic contour (at $x / R=0.185$ along $y=0$ ). It has been scaled by the theoretical estimate $\psi_{T}$ from (4.22a). For both cyclonic and anticyclonic forcing $T_{I} / \psi_{I}$ is always less than about one. Cyclonic and anticyclonic forcing transports agree for moderate nonlinearity $\delta_{I} / \delta_{M}<1.5$, but diverge as the nonlinearity increases. The anticyclonic transports (both $T_{I}$ and $T_{T}$ ) increase smoothly and appear to plateau for $\delta_{I} / \delta_{M}>2.5$. While the cyclonic transports steadily decrease. The anticyclonic transport ratio, $T_{T} / T_{I}$, is approximately 0.8 independent of $\delta_{I} / \delta_{M}$. The average cyclonic ratio is slightly larger, $\approx 0.85$, and increases weakly with the nonlinearity. The experimental transports are lower and the ratio slightly larger than the numerical results of Figure 13. However, the overall agreement in the anticyclonic case is quite good.

\section{Conclusions and discussion}

Although the circulation around planetary scale islands has its own intrinsic fascination, its importance oceanographically is connected to its role in determining the exchange 
between adjacent basins. The by now classical example is the flow around Australia which, as Godfrey (1989) pointed out, is an essential element in the global meridional overturning circulation.

Earlier theoretical studies have described the dynamics of flow around islands with vertical side boundaries. In this paper we have emphasized the important changes that take place when strong, encircling topographic slopes are considered. In particular, the appearance of closed, ambient potential vorticity contours, $f / h$ has been shown to trap fluid in a resonant geostrophic mode with flow following $q=f / h$ contours and whose amplitude, under wind stress forcing, is limited only by dissipation as described by (4.16). It also follows that the value of the barotropic stream function on the island, i.e. the Island Constant $\Psi_{I}$, is probably not the best measure of the inter-basin mass exchange since a portion of that flux is trapped over the $q$ contours. The value of the stream function on the outermost closed contour, $\psi_{T}$, is likely a better measure of the true inter-basin exchange. In the linear limit, $\psi_{T}$ closely agrees with the island constant obtained for an island whose shape is determined by that outermost contour.

Since both constants are determined by what are, in reality, vorticity budgets in domains with open boundaries, it is not surprising that these measures of the fluxes, both trapped and inter-basin, depend on the degree of nonlinearity of the flow which is responsible for changes in the flow structure. Both numerical (Fig. 13) and laboratory experiments (Fig. 27) show similar enhancements of both $\Psi_{I}$ and $\psi_{T}$ with respect to the linear theoretical results of Section 4. We have not yet determined the mechanism in detail for the nonlinear enhancement; is most likely due to a combination of causes. The enhancement in the range $1<\delta_{I} / \delta_{M}<2$, where the flow is steady, must be due to the alteration of the steady flow structure and the increasing north-south asymmetry. Larger nonlinearity, where the flow becomes unstable and eddy-rich, may have important contributions to the vorticity budgets in the domains used in the linear theory by eddy fluxes. Describing that mechanism more clearly is a subject for future work.

The presence of the topographic skirt imposes an asymmetry between cyclonic and anticyclonic wind stress forcing such that the threshold for the appearance of timedependent eddy fields is considerably raised in the cyclonic case. This is another example (see LaCasce et al., 2008) where the direction of propagation of topographic waves, either with or against the wind-driven flow, strongly affects its transition to time dependence.

A robust feature of the flow structure over a broad range of forcing, i.e. over a range of $\delta_{I} / \delta_{M}$ is the western meander appearing at the western edge of the skirt where the flow, driven along the $f / h$ contours on the skirt leaves the skirt and its topographic control and blends into the Sverdrup interior west of the skirt. The abruptness of the trajectory change is due to the rapid change in the direction of the $f / h$ contours and has been observed in both our numerical and laboratory work. In cases such as the one examined here where the topography is steep, the change in direction of the flow becomes dramatically abrupt.

Since the effect of topography is so dramatic in altering the circulation around the island we have considered, it seems of interest to also examine in the future what role density 
stratification might play. For an island with vertical walls the barotropic mode, yielding the vertical average circulation, remains essentially unchanged in the presence of stratification. The presence of topography mixes the barotropic and baroclinic modes of motion and it remains to be seen how the joint effect of topography and stratification (JETS) will affect the transport between basins as well as the vertical structure of the exchange. As we have pointed out earlier, the identification of the outermost closed $q$ contour with the island shape satisfying the Island Rule could be fundamentally altered in a more turbulent regime as might occur in a baroclinic ocean with a wider range of instabilities. We expect to examine that possibility in a future study.

Finally, in our present configuration the dynamics of the flow near the island is strongly coupled to the dynamics of the basin's western boundary layer, most strongly in the exit region of the boundary current. This is especially important for the development of time-dependent motion. This coupling is inescapable if we use an island extended far enough in the north-south direction to block both the eastern and western streamlines of the gyre's Sverdrup flow. Given the nature of the forcing we have used (constant wind stress curl) the variation of the Sverdrup stream function in latitude is determined entirely by the curvature of the eastern boundary. It would be interesting to introduce different forcing, also reproducible in the laboratory, that would allow the island to experience both eastward and westward flow while at the same time being well separated from the intricate flow behavior of the western boundary current. Such possibilities are being examined.

Acknowledgements. This work was supported in part by NSF grant OCE-0451086 (JP) and NSF OCE 05-25729 (KH).

\section{REFERENCES}

Beardsley, R. C. 1969. A laboratory model of the wind-driven ocean circulation. J. Fluid Mech., 26, $\underline{255-271 .}$

Blumberg, A. F. and G. L. Mellor. 1987. A description of a three-dimensional coastal ocean circulation model, in Three-Dimensional Coastal Ocean Models, N. Heaps, ed., Am. Geophys. Union, 208 pp.

Firing, E., B. Qiu and W. Miao. 1999. Time-dependent Island Rule and its application to the time-varying North Hawaiian ridge current. J. Phys. Oceanogr., 29, 2671-2688.

Godfrey, J. S. 1989. A Sverdrup model of the depth-integrated flow from the world ocean allowing for island circulations. Geophys. Astrophys. Fluid Dyn., 45, 89-112.

Griffiths, R. W. and A. E. Kiss. 1999. Flow regimes in a wide "sliced-cylinder" model of homogeneous beta-plane circulation. J. Fluid Mech., 399, 205-235.

Helfrich, K. R., J. Pedlosky and E. Carter. 1999. The shadowed island. J. Phys. Oceanogr., 29, $\underline{2559-2577 .}$

Huang, R. X. and X. Jin. 2002. Deep circulation in the South Atlantic induced by bottom-intensified mixing over the midocean ridge. J. Phys. Oceanogr., 32, 1150-1164.

Joyce, T. M. and A. Proshutinsky. 2007. Greenland's Island Rule and the Arctic Ocean Circulation. J. Mar. Res., 65, 639-653.

Katsman, C. A. 2006. Impacts of localized mixing and topography on the stationary abyssal circulation. J. Phys. Oceanogr., 36, 1660-1671.

Kiss, A. E. 2002. Potential vorticity "crises", adverse pressure gradients, and western boundary current separation. J. Mar. Res., 60, 779-803. 
LaCasce, J. H., O. A. Nøst and P. E. Isachsen. 2008. Asymmetry of free circulations in closed ocean gyres. J. Phys. Oceanogr., 38, 517-526.

Meacham, S. P. and P. S. Berloff. 1997. Instabilities of a steady, barotropic, wind driven circulation. J. Mar. Res., 55, 885-913.

Mellor, G. L. 1992. User's guide for a three-dimensional, primitive equation, numerical ocean model, Program in Atmospheric and Oceanic Sciences, Princeton University, 35 pp.

Nof, Doron. 2000. Does the wind control the import and export of the South Atlantic? J. Phys. Oceanogr., 30, 2650-2667.

Pedlosky, J. and H. P. Greenspan. 1967. A simple laboratory model for the oceanic circulation. J. Fluid Mech., 27, 291-304.

Pedlosky, J., L. J. Pratt, M. A. Spall and K. R. Helfrich. 1997. Circulation around islands and ridges. J. Mar. Res., 55, 1199-1251.

Salmon, R. 1992. A two-layer Gulf Stream over a continental slope. J. Mar. Res., 50, 341-365.

Stommel, H. and A. B. Arons. 1960. On the abyssal circulation of the world ocean-I. Stationary planetary flow patterns on a sphere. Deep-Sea Res., 6, 140-154.

Received: 22 June, 2009; revised: 23 September, 2009. 